\title{
Impact of deltamethrin selection on $k d r$ mutations and insecticide detoxifying enzymes in Aedes aegypti from Mexico
}

Yamili Contreras-Perera' ', Gustavo Ponce-Garcia' ${ }^{1}$ Karina Villanueva-Segura' ${ }^{1}$ Beatriz Lopez-Monroy', Iram P. Rodríguez-Sanchez ${ }^{1}$, Audrey Lenhart ${ }^{2}$, Pablo Manrique-Saide ${ }^{3}$ and Adriana E. Flores ${ }^{1 *}$ (D)

\begin{abstract}
Background: Insecticide resistance is a serious problem for vector control programmes worldwide. Resistance is commonly attributed to mutations at the insecticide's target site or increased activity of detoxification enzymes.

Methods: We determined the knockdown concentration $\left(\mathrm{KC}_{50}\right)$ and lethal concentration $\left(\mathrm{LC}_{50}\right)$ of deltamethrin in six natural populations of adult Aedes aegypti from southeastern Mexico. These populations were then selected over five generations using the $\mathrm{LC}_{50}$ from the preceding generation that underwent selection, and the heritability of deltamethrin resistance was quantified. For each generation, we also determined the frequency of the $k d r$ alleles $L 410$, 11016 and C1534, and the levels of activity of three enzyme families ( $\alpha$ - and $\beta$-esterases, mixed-function oxidases and glutathione S-transferases (GST)) associated with insecticide detoxification.

Results: There was an increase in $\mathrm{KC}_{50}$ and $\mathrm{LC}_{50}$ values in the subsequent generations of selection with deltamethrin $\left(F_{S 5} V s F_{S 0}\right)$. According to the resistance ratios (RRs), we detected increases in $\mathrm{LC}_{50}$ ranging from 1.5 to 5.6 times the values of the parental generation and in $\mathrm{KC}_{50}$ ranging from 1.3-3.8 times the values of the parental generation. Triple homozygous mutant individuals (tri-locus, LL/II/CC) were present in the parental generations and increased in frequency after selection. The frequency of $\mathrm{L} 410$ increased from 1.18-fold to 2.63-fold after selection with deltamethrin $\left(F_{S 5}\right.$ VS $\left.F_{S O}\right)$ in the populations analyzed; for 11016 an increase between 1.19-fold to 2.79-fold was observed, and C1534 was fixed in all populations after deltamethrin selection. Enzymatic activity varied significantly over the generations of selection. However, only a- esterase activity remained elevated in multiple populations after five generations of deltamethrin selection. We observed an increase in the mean activity levels of GSTs in two of the six populations analyzed.
\end{abstract}

Conclusions: The high levels of resistance and their association with high frequencies of $k d r$ mutations (V410L, V1016l and F1534C) obtained through artificial selection, suggest an important role of these mutations in conferring resistance to deltamethrin. We highlight the need to implement strategies that involve the monitoring of $k d r$ frequencies in insecticide resistance monitoring and management programmes.

Keywords: Aedes aegypti, Heritability, kdr mutations, V410L, V1016I, F1534C, Metabolic detoxification

*Correspondence: adriana.floressr@uanl.edu.mx

1 Facultad de Ciencias Biologicas, Universidad Autonoma de Nuevo Leon,

Cd, Universitaria, San Nicolas de los Garza, N.L. CP. 66455, Mexico

Full list of author information is available at the end of the article

c) The Author(s) 2020. This article is licensed under a Creative Commons Attribution 4.0 International License, which permits use, sharing, adaptation, distribution and reproduction in any medium or format, as long as you give appropriate credit to the original author(s) and the source, provide a link to the Creative Commons licence, and indicate if changes were made. The images or other third party material in this article are included in the article's Creative Commons licence, unless indicated otherwise in a credit line to the material. If material is not included in the article's Creative Commons licence and your intended use is not permitted by statutory regulation or exceeds the permitted use, you will need to obtain permission directly from the copyright holder. To view a copy of this licence, visit http://creativeco mmons.org/licenses/by/4.0/. The Creative Commons Public Domain Dedication waiver (http://creativecommons.org/publicdomain/ zero/1.0/) applies to the data made available in this article, unless otherwise stated in a credit line to the data. 


\section{Background}

Aedes aegypti (L.) is an arbovirus vector of great public health importance, and is the principal vector transmitting dengue, chikungunya and Zika viruses in the Americas [1-3]. Currently, there are no widely licensed vaccines or specific treatments for any of these diseases, rendering vector control the principal strategy for preventing their transmission. The reduction or elimination of larval habitats and chemical control with the use of larvicides and adulticides are the main strategies to reduce vector-human contact and break the transmission cycles of these diseases [4]. The insecticides used for such purposes worldwide are mainly from the following chemical groups: organophosphates, organochlorines, carbamates and pyrethroids $[5,6]$. Pyrethroids are often the first line of defense to break the transmission of these diseases, and they are the most commonly used worldwide due to their low toxicity to mammals including humans $[7,8]$.

In Mexico, the application of pyrethroid insecticides is regulated by the Ministry of Health, and their use dates back to 1999 when the use of DDT was banned in the health sector. At this time, permethrin became the insecticide of choice for space spraying and deltamethrin for indoor residual spraying [9]. In 2011, the use of permethrin was discontinued; however, deltamethrin remained on the list of insecticides approved for use as an adulticide in indoor residual spraying. In 2012 other pyrethroids were added to the list; however, deltamethrin remained, and in 2016 its use for space spraying applications was also approved, a practice that remains to date [10]. The use of pyrethroids in Mexico has been favored for more than 20 years, which led to widespread resistance in mosquito populations [11-13].

Insecticide resistance is one of the main obstacles to the success of vector control programmes [12, 14, 15]. Two of the principal mechanisms that cause resistance in mosquitoes are alterations at the target site of the insecticide, including knockdown resistance $(k d r)$ mutations on the voltage-gated sodium channel gene $(v g s c)$, and increased metabolic activity $[16,17]$. Targetsite resistance arising from $k d r$ mutations can cause resistance to both DDT and pyrethroids, since both types of insecticides target sodium channels at the axon level [18].

Since the discovery of the L1014F mutation in Musca domestica and since the determination of its role in insecticide resistance [19], more than 50 mutations have been described for the $v g s c$ gene in different pests and disease vectors [20]. Thirteen of these sequence changes are commonly found in Ae. aegypti in ten different positions covering five regions of the vgsc gene (IIS4-5, IIS5-6, IIS-6, IIIS6 and IVS5), identified at positions $410(\mathrm{~V} \rightarrow \mathrm{L}), 419(\mathrm{~V} \rightarrow \mathrm{L}), 923(\mathrm{G} \rightarrow \mathrm{I}), 982$
$(\mathrm{L} \rightarrow \mathrm{W}), 989(\mathrm{~S} \rightarrow \mathrm{P}), 1011(\mathrm{I} \rightarrow \mathrm{M}$ or $\mathrm{V}), 1016(\mathrm{~V} \rightarrow \mathrm{G}$ or I), $1520(\mathrm{~T} \rightarrow \mathrm{I}), 1534(\mathrm{~F} \rightarrow \mathrm{C}$ or $\mathrm{L})$, and $1763(\mathrm{D} \rightarrow \mathrm{Y})$ [21-29]. It should be noted that only five of the mutations described for this species have been functionally associated with sensitivity to pyrethroids and DDT by heterologous expression and electrophysiology assays; only S989P, I1011M, V1016G, F1534C and V410L have been confirmed [20, 27, 30,31]. Similarly, it is important to note that mutations are usually associated with a specific geographic sector, for example, mutation V1016G has been described in Southeast Asia but not in the Americas [32].

In Mexico, mutations F1534C, V1016I and V410L have been reported in populations of Ae. aegypti in different states, sites where their temporal distribution indicates the absence of mutant alleles in 2000, with a gradual increase in these mutations during the period of 20022008 , up to fixation of the mutant alleles in 2016, showing an allelic frequency for the three simultaneous changes of 0.47 [30]. Of these mutations, the change from phenylalanine to cysteine at position 1534 was described initially by [25], who associated its occurrence with pyrethroid resistance; however, confirmation by heterologous expression and electrophysiology has demonstrated that this change reduces sensitivity of VGSC to type I but not type II pyrethroids [33]. The presence of this mutation has been linked to the development of low resistance levels and its association with other gene changes to the establishment of a more resistant phenotype. Such is the case of its association with V1016I, described by Saavedra et al. [22], where its participation in the reduction of sensitivity of VGSC has not been identified when appearing as a single mutation [31]. An analysis of the frequency of V1016I and F1534C in 24 populations of Ae. aegypti in Mexico showed that the most likely evolutionary process is the appearance of F1534C and then V1016I, this being corroborated by the low fitness of haplotype I1016/F1534 [34] and the increase in insecticide resistance expressed in Xenopus oocytes when inducing the two mutations [31]. Temporal distribution studies of this mutation in Mexico identified an increase in the frequency of the I1016 mutant allele during the period of 1996 to 2000 with a frequency of $0.04 \%$ up to an increase to $33.2 \%$ in 2007 to 2009 [35].

The V410L mutation has been recently described by Haddi et al. [27], who determined their association with resistance to type I and II pyrethroids. This mutation has been studied in Mexico in populations of Ae. aegypti collected between 2000 and 2016 where its appearance was reported in 2002 in heterozygous individuals collected in Coatzacoalcos, Veracruz, and where the increase in the frequency of L410 was also documented as being as high as 0.9 in populations collected in 2014 in Merida, 
Yucatan [30]. A similar pattern was found for $26 \mathrm{popu}-$ lations of Ae. aegypti collected in the eastern part of Mexico, showing a frequency of the L410 allele close to the one in populations from Minatitlan and Jose Cardel in Veracruz and Cancun in Quintana Roo $(0.99,0.97$ and 0.93, respectively), but the average values, as well as the interval of frequencies observed in populations ranged from 0.3 to 0.99 [36].

Metabolic resistance to pyrethroids is mediated mainly by glutathione $\mathrm{S}$-transferases (GSTs), esterases and mixed-function oxidases (MFOs) [37-39]. In Mexico, Flores et al. [14] previously reported high levels of $\alpha$ and $\beta$-esterase activity related to permethrin selection in populations of Ae. aegypti from Baja California Norte and Sur. The increased activity of these enzymes has also been reported in populations from the state of Sonora in a study of permethrin resistance and in the state of

Table 1 Collection sites, geographical coordinates

\begin{tabular}{llll}
\hline State & Municipality & Location & Coordinates \\
\hline Yucatan & Merida & Manzana 115 & $20^{\circ} 56^{\prime} 42^{\prime \prime} \mathrm{N}, 89^{\circ} 38^{\prime} 36^{\prime \prime} \mathrm{W}$ \\
& Progreso & & $21^{\circ} 16^{\prime} 52^{\prime \prime} \mathrm{N}, 89^{\circ} 39^{\prime} 54^{\prime \prime} \mathrm{W}$ \\
& Hunucma & & $21^{\circ} 00^{\prime} 58^{\prime \prime} \mathrm{N}, 89^{\circ} 52^{\prime} 38^{\prime \prime} \mathrm{W}$ \\
& Hoctun & & $20^{\circ} 51^{\prime} 50^{\prime \prime} \mathrm{N}, 89^{\circ} 12^{\prime} 03^{\prime \prime} \mathrm{W}$ \\
Veracruz & Agua dulce & Agua dulce & $18^{\circ} 08^{\prime} 33^{\prime \prime} \mathrm{N}, 94^{\circ} 08^{\prime} 36^{\prime \prime} \mathrm{W}$ \\
& La Antigua & Jose Cardel & $19^{\circ} 22^{\prime} 15^{\prime \prime} \mathrm{N}, 96^{\circ} 22^{\prime} 35^{\prime \prime} \mathrm{W}$ \\
\hline
\end{tabular}

Veracruz in a study of chlorpyrifos resistance [40, 41]. A further study reported high levels of GST activity in the state of Guerrero, which was found to be related to resistance to DDT [12].

Given the background levels of pyrethroid and DDT resistance in many parts of Mexico, the extent to which ongoing insecticide pressure selects for specific mechanisms of resistance is not well understood. To better understand how insecticide exposure selects for resistance we evaluate the effect of laboratory selection with deltamethrin on the underlying resistance mechanisms in populations of Ae. aegypti from southeastern Mexico, over five generations of selection.

\section{Methods}

\section{Biological material}

Eggs and larvae of Ae. aegypti were collected during 2014 from six sites: Merida, Progreso, Hunucma, and Hoctun in the State of Yucatan; and Agua Dulce and Jose Cardel, in the State of Veracruz (Table 1, Fig. 1). The criteria for site collection were: high incidence of dengue and frequent application of insecticides for its control; high infestation index of homes with Ae. aegypti; and previous registration of insecticide resistance. The collection of the entomological material was carried out in peridomestic sites. In each, entomological inspections were performed for the collection of stages of Ae. aegypti in at least 20 potential breeding places per site, such as containers, tires and flowerpots.

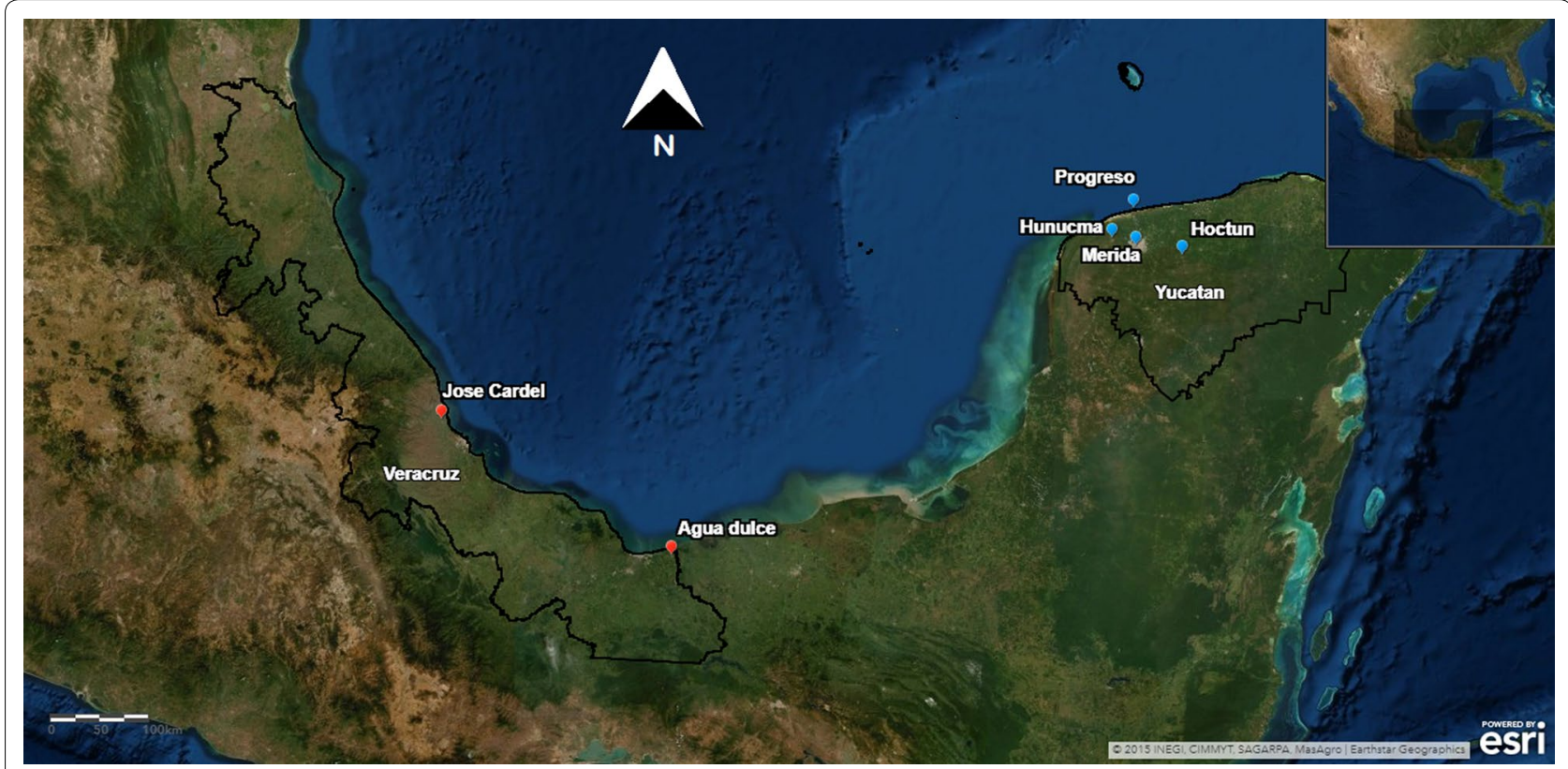

Fig. 1 Collection sites of Aedes aegypti from Veracruz and Yucatan states from Mexico 
The biological material obtained from the field was reared under insectary conditions at $26 \pm 2{ }^{\circ} \mathrm{C}$ and $70-80 \%$ relative humidity with a $12 \mathrm{~h}: 12 \mathrm{~h}$ (light:dark) photoperiod. The adults obtained from field-collected material were allowed to intermate, and the eggs resulting were designated $\mathrm{F}_{\mathrm{S} 0}$ (without previous selection). The New Orleans strain (NO) was used as a susceptible reference in the study, this strain was originally obtained from the CDC (Atlanta, GA, USA) and has been maintained since 2002.

\section{Bioassays}

The bioassays consisted of exposing 20-25 non-bloodfed, 2-3-day-old $\mathrm{F}_{\mathrm{S} 0}$ female mosquitoes to different concentrations of the pyrethroid deltamethrin (>98\% purity; Chem Service, West Chester, PA, USA) based on the bottle bioassay methodology described by the CDC [42]. Each bioassay consisted of bottles containing different concentrations of deltamethrin resulting in mortalities between 9-90\%, with at least three replicates for each concentration, and an untreated control bottle. The numbers of knocked-down mosquitoes were recorded at $1 \mathrm{~h}$. After $1 \mathrm{~h}$ of exposure, all mosquitoes were gently transferred to a recovery container without insecticide and were offered a cotton ball soaked in a sugar solution. Mortality was recorded at $24 \mathrm{~h}$. Both the bottles and recovery containers were kept at $24 \pm 2{ }^{\circ} \mathrm{C}$ and $70 \% \mathrm{RH}$.

Rates of $k d r$ and $24 \mathrm{~h}$ recovery were analyzed using a logistic regression model, QCal (https://sourceforge.net/ projects/irmaproj/files/Qcal/) [43]. We determined the $\mathrm{KC}_{50}$ (concentration causing 50\% knockdown) after 1 $\mathrm{h}$ of exposure. The $\mathrm{LC}_{50}$ (concentration causing $50 \%$ mortality) was estimated from mortality data $24 \mathrm{~h}$ after recovery. The confidence intervals were calculated using a significance level of $\alpha=0.05$. The mortalities were corrected according to Abbott's formula [44] when mortality was observed in the control bottles. The resistance ratios (RR) were calculated by dividing the $\mathrm{KC}_{50}$ or $\mathrm{LC}_{50}$ by the $\mathrm{KC}_{50}$ or $\mathrm{LC}_{50}$ of the susceptible New Orleans (NO) Ae. aegypti reference strain. The magnitude of resistance was classified as high ( $R R>10$-fold), moderate ( $R R$ between 5-10-fold) or low ( $R R<5$-fold) according to the criteria proposed by Mazarri \& Georghiou [45].

\section{Selection with deltamethrin}

Selection cohorts were generated according to the methodology described by Saavedra et al. [46]. The selection was carried out exposing 650-1000 $\mathrm{F}_{\mathrm{S} 1}$ males and females (1:4) for $1 \mathrm{~h}$ to the $\mathrm{LC}_{50}$ obtained for the previous generation $\left(\mathrm{F}_{\mathrm{S} 0}\right)$ by the method described above. The survivors at $24 \mathrm{~h}$ were transferred to mosquito cages for breeding the subsequent generation $\left(\mathrm{F}_{\mathrm{S} 2}\right)$. The dose response parameters were calculated again for the $F_{S 1}$ cohort and were used to select the next generation $\left(\mathrm{F}_{\mathrm{S} 2}\right)$. This procedure was repeated for each generation of selection until $\mathrm{F}_{\mathrm{S} 5}$. The New Orleans strain was tested at the same time as the $\mathrm{F}_{\mathrm{S} 0}$ and was not tested in each generation of selection.

$\mathrm{KC}_{50}$ and $\mathrm{LC}_{50}$ values were compared between generations to monitor changes in resistance. The $\mathrm{KC}_{50}$ and $\mathrm{LC}_{50}$ values were considered significantly different if their 95\% confidence intervals did not overlap.

\section{Realized heritability}

Resistance risk assessment was made by calculating realized heritability values $\left(h^{2}\right)$ in all selection cohorts for the knockdown $\left(\mathrm{KC}_{50}\right)$ and lethal $\left(\mathrm{LC}_{50}\right)$ parameters as described by Tabashnik [47]. The heritability index $\left(\mathrm{h}^{2}=\mathrm{R} / \mathrm{S}\right)$ is calculated as the ratio of the response to selection (R) to the selection differential (S) according to the artificial selection technique of Falconer [48] and is related to the additive genetic variance for a trait. A low $\mathrm{h}^{2}$ predicts no additive genetic variance for a trait and a poor or very slow response to artificial selection, while a high $h^{2}$ predicts a large additive genetic variance at one or a few loci that govern a trait and predicts a rapid response to artificial selection.

The selection differential is expressed as the product of selection intensity (i) and phenotypic standard deviation $(S=i \sigma)$. The response to selection was determined for each generation as the difference in population means between subsequent generations with the probit analysis: $\mathrm{R}=\log \left(\right.$ Final $\mathrm{KC}_{50}$ or $\left.\mathrm{LC}_{50}\right)-\log$ (Initial $\mathrm{KC}_{50}$ or $\left.\mathrm{LC}_{50}\right) / \mathrm{n}$, where final $\mathrm{KC}_{50}$ or $\mathrm{LC}_{50}$ area the values of the offspring after $\mathrm{n}$ generations of selection and initial $\mathrm{KC}_{50}$ or $\mathrm{LC}_{50}$ are the values of the parental generation before $n$ generations of selection. The selection intensity was calculated from the proportion of surviving individuals in the examined population. The difference between $\mathrm{KC}_{50}$ or $\mathrm{LC}_{50}$ was calculated on a logarithmic scale because the logarithm of tolerance was assumed to be normally distributed. The phenotypic standard deviation at $\mathrm{t}$-th generation $\left(\sigma_{t}\right)$ was obtained as the inverse of the regression slope: $\sigma_{\mathrm{t}}=1 / \mathrm{b}_{\mathrm{t}}$. The parameters $(R, i$ and $\sigma)$ were determined at every generation and the realized heritability was estimated as the regression coefficient of cumulative responses on cumulative selection differentials [48].

\section{Molecular assays}

DNA was extracted from individual mosquitoes by the technique described by Coen et al. [49], and the DNA pellet was resuspended in $30 \mu \mathrm{l}$ of ultrapure molecular grade water (Corning Cellgro ${ }^{\mathrm{TM}}$, Manassas, VA, USA). The quantity and quality of DNA were determined using a NanoDrop spectrophotometer 2000 (Thermo Fisher Scientific, Woonsocket, RI, USA). 
The $k d r$ alleles $1534 \mathrm{C}$, 1016I and 410L were detected by endpoint PCR using a Bio-Rad (Hercules, CA, USA) T100TM thermocycler using the primers for each of the mutations described by Saavedra et al. [22, 30] and Yanola et al. [25].

The primers used to detect the V1016I mutation were: V1016fw (5'-GCG GGC AGG GCG GCG GGG GCG GGG CCA CAA ATT GTT TCC CAC CCG CAC CGG-3'); I1016fw (5'-GCG GGC ACA AAT TGT TTC CCA CCC GCA CTG A-3'); and I1016R (5'-TGA TGA ACC SGA ATT GGA CAA AAG $C-3^{\prime}$ ). The PCR was carried out in a reaction mixture of $12.5 \mu$ l containing $1.25 \mu$ of $10 \times$ buffer (Invitrogen, Carlsbad, CA, USA), $1.5 \mathrm{mM} \mathrm{MgCl}{ }_{2}, 0.2 \mathrm{mM}$ dNTPs, $5 \mu \mathrm{M}$ of each primer, $100 \mathrm{ng}$ genomic DNA, and $2 \mathrm{U}$ Taq polymerase (Invitrogen). The PCR reaction conditions were: $95^{\circ} \mathrm{C}$ for 5 min; 29 cycles of $95{ }^{\circ} \mathrm{C}$ for $1 \mathrm{~min}, 60^{\circ} \mathrm{C}$ for $1 \mathrm{~min}, 72{ }^{\circ} \mathrm{C}$ for $1 \mathrm{~min} 15 \mathrm{~s}$; and a final extension step at $72{ }^{\circ} \mathrm{C}$ for 10 $\min [22]$.

The primers used to detect the F1534C mutation were: C1534fw (5'-GCG GGC AGG GCG GCG GGG GCG GGG CCT CTA CTT TGT GTT CTT CAT CAT GTG3'); F1534fw (5'-GCG GGC TCT ACT TTG TGT TCT TCA TCA TAT T-3'); and F1534R (5'-TCT GCT CGT TGA AGT TGT CGA T-3'). The PCR was carried out in a reaction mixture of $12.5 \mu \mathrm{l}$ containing $1.25 \mu \mathrm{l}$ of $10 \times$ buffer (Invitrogen), $1.5 \mathrm{mM} \mathrm{MgCl}_{2}, 0.1 \mathrm{mM}$ dNTPs, 0.5 $\mu \mathrm{M}$ of each primer, $100 \mathrm{ng}$ genomic DNA, and $1 \mathrm{U}$ Taq polymerase (Invitrogen). The PCR reaction conditions were: $95^{\circ} \mathrm{C}$ for $4 \mathrm{~min}$; 35 cycles of $95^{\circ} \mathrm{C}$ for $1 \mathrm{~min}, 57^{\circ} \mathrm{C}$ for $1 \mathrm{~min}, 72^{\circ} \mathrm{C}$ for $1 \mathrm{~min}$; and a final extension step at $72{ }^{\circ} \mathrm{C}$ for $4 \mathrm{~min}$ [50].

The primers used to detect the V410L mutation were: V410fw (5'-GCG GGC AGG GCG GCG GGG GCG GGG CCA TCT TCT TGG GTT CGT TCT ACC GTG$3^{\prime}$ ), L410fw (5'-GCG GGC ATC TTC TTG GGT TCG TTC TAC CAT T-3'); and 410R (5'-TTC TTC CTC GGC GGC CTC TT-3'). Amplification was performed following the methodology described by VillanuevaSegura et al. [36]. In a $1.5 \mathrm{ml}$ tube, a master mix was prepared as follows: $12.50 \mu \mathrm{l}$ of GoTaq (Promega, Madison, WI, USA) was mixed with $11.35 \mu \mathrm{l}$ of nuclease-free $\mathrm{H}_{2} \mathrm{O}$ (NFW, Promega), and $1 \mu \mathrm{M}$ of each of the primers (V410fw, L410fw and 410rev) was then added. The master mix $(24 \mu \mathrm{l})$ was then placed in $0.2 \mathrm{ml}$ stoppered tubes followed by $1 \mu \mathrm{l}$ of mosquito genomic DNA ( 25 $\mathrm{ng}$ ). The tubes were centrifuged for $1 \mathrm{~min}$ at $3300 \times \mathrm{rpm}$, then placed in a T100 thermocycler (Bio-Rad) with the following temperature program: $3 \mathrm{~min}$ at $95^{\circ} \mathrm{C} ; 30$ cycles of $1 \mathrm{~min}$ at $95^{\circ} \mathrm{C}, 20 \mathrm{~s}$ at $56^{\circ} \mathrm{C}$ and $20 \mathrm{~s}$ at $72{ }^{\circ} \mathrm{C}$; followed by 5 min at $72{ }^{\circ} \mathrm{C}$.
DNA from the New Orleans susceptible strain was used as a negative control for the $k d r$ assays, and previously genotyped individuals were used as positive controls.

The PCR products for the V410L, V1016I and F1534C assays were visualized on $2.5 \%, 3 \%$ and $4 \%$ agarose gels, respectively, using an UVITEC (Cambridge, UK) imaging system.

The frequencies of the alleles were determined for each population at each generation. We verified that the populations at the parental generation $\left(\mathrm{F}_{\mathrm{S} 0}\right)$ were in HardyWeinberg equilibrium by means of Chi-square analysis. In addition, Wright's $\mathrm{F}_{\mathrm{IS}}$ inbreeding coefficient was estimated, along with Wald's correction $[51,52]$.

\section{Biochemical assays}

Thirty-two 2-4-day-old unfed female mosquitoes were individually homogenized in $2 \mathrm{ml}$ of $0.01 \mathrm{M}$ phosphate buffer ( $\mathrm{pH} 7.2$ ). Aliquots of $100 \mu \mathrm{l}$ of the homogenate were distributed in triplicate in flat-bottom microplates (Corning, Tewksbury, MA, USA) for testing the activity levels of the following families of enzymes: $\alpha$ - and $\beta$-esterases, MFOs, and GSTs, based on mosquito-specific biochemical assay protocols [53-56].

\section{$a$ - and $\beta$-esterases}

To measure the activity levels of $\alpha$ - and $\beta$-esterases, 100 $\mu \mathrm{l}$ of either $\alpha$ - or $\beta$-naphthyl acetate (Sigma-Aldrich, St. Louis, MO,USA) dissolved in acetone (CTR Scientific, Monterrey, N.L., Mexico), and phosphate buffer $\left(\mathrm{KPO}_{4}\right.$, $\mathrm{pH}$ 7.2) was added to each well and incubated for $20 \mathrm{~min}$ at room temperature. Subsequently, $100 \mu \mathrm{l}$ of fast blue (tetrazotized O-dianisidine (Sigma-Aldrich) dissolved in distilled water) was added to each well and the microplates incubated for another $4 \mathrm{~min}$, before absorbance was read at $540 \mathrm{~nm}$ using a spectrophotometer (ASYS Hitech $\mathrm{GmbH}$, Eugendorf, Austria).

\section{MFOs}

$200 \mu \mathrm{l}$ of TMBZ (3,3,5,5-tetramethyl-benzidine dihydrochloride (Sigma-Aldrich) previously dissolved in methanol (Jalmek, Monterrey, N.L. Mexico) and 0.25 M sodium acetate buffer $\mathrm{pH}$ 5.0) were added to each well. Subsequently $25 \mu \mathrm{l}$ of $3 \%$ hydrogen peroxide $\left(\mathrm{H}_{2} \mathrm{O}_{2}\right)$ (Jalmek) was added to each well. After $10 \mathrm{~min}$ of incubation at room temperature the microplate was read at a wavelength of $620 \mathrm{~nm}$.

\section{GSTs}

$100 \mu \mathrm{l}$ of reduced glutathione (GSH; Sigma-Aldrich) and $100 \mu \mathrm{l}$ 1-chloro-2,4 dinitrobenzene (CDNB; SigmaAldrich) previously diluted in acetone and $\mathrm{KPO}_{4}$ buffer were added to each well. Absorbance was read at $340 \mathrm{~nm}$ immediately (T0) and after $10 \mathrm{~min}$ of incubation (T10). 
Table 2 Deltamethrin knockdown concentrations $\left(\mathrm{KC}_{50}\right.$ and $\left.\mathrm{KC}_{90}\right)$ and resistance ratios (RR) for Aedes aegypti females in the parental generation $\left(\mathrm{F}_{\mathrm{SO}}\right)$ and the deltamethrin-selected generations $\left(\mathrm{F}_{\mathrm{S} 1}-\mathrm{F}_{\mathrm{S} 5}\right)$

\begin{tabular}{|c|c|c|c|c|c|c|c|c|}
\hline \multirow[t]{2}{*}{ Population } & \multirow[t]{2}{*}{ Generation } & \multirow[t]{2}{*}{$\mathrm{n}^{\mathrm{a}}$} & \multicolumn{4}{|l|}{$K C^{b} \mu g / B$} & \multicolumn{2}{|l|}{$\mathrm{RR}^{\mathrm{c}}$} \\
\hline & & & $\mathrm{KC}_{50}(\mathrm{Cl})$ & $\mathrm{KC}_{90}(\mathrm{Cl})$ & $b^{d} \pm S E$ & $P$-value & $\mathrm{KC}_{50}$ & $\mathrm{KC}_{90}$ \\
\hline \multirow[t]{6}{*}{ Merida } & $\mathrm{F}_{\mathrm{SO}}$ & 345 & $1.85(1.69-2.03)$ & $3.89(3.27-4.63)$ & $2.95(0.35)$ & $<0.001$ & 20 & 8 \\
\hline & $\mathrm{F}_{\mathrm{S} 1}$ & 403 & $1.62(1.45-1.82)$ & $4.65(3.62-5.97)$ & $2.09(0.21)$ & $<0.001$ & 18 & 10 \\
\hline & $\mathrm{F}_{\mathrm{S} 2}$ & 525 & $1.85(1.63-2.09)$ & $6.75(4.88-9.33)$ & $1.69(0.19)$ & $<0.001$ & 20 & 14 \\
\hline & $\mathrm{F}_{\mathrm{S3}}$ & 540 & $2.42(2.04-2.85)$ & $15.19(14.58-21.80)$ & $1.19(0.10)$ & $<0.001$ & 27 & 33 \\
\hline & $\mathrm{F}_{\mathrm{S4}}$ & 440 & $2.55(2.17-3.00)$ & $12.79(9.01-18.16)$ & $1.36(0.13)$ & $<0.001$ & 28 & 27 \\
\hline & $\mathrm{F}_{\mathrm{S5}}$ & 400 & $3.44(2.93-4.04)$ & 14.84 (10.88-20.23) & $1.50(0.15)$ & $<0.001$ & 38 & 32 \\
\hline \multirow[t]{6}{*}{ Progreso } & $\mathrm{F}_{\mathrm{SO}}$ & 490 & $0.54(0.45-0.64)$ & $3.22(2.26-4.60)$ & $1.23(0.11)$ & $<0.001$ & 6 & 7 \\
\hline & $\mathrm{F}_{\mathrm{S} 1}$ & 526 & $0.67(0.60-0.79)$ & $3.00(2.25-3.99)$ & $1.50(0.13)$ & $<0.001$ & 7 & 7 \\
\hline & $\mathrm{F}_{\mathrm{S2}}$ & 417 & $1.78(1.53-2.07)$ & $7.13(4.84-10.50)$ & $1.58(0.18)$ & $<0.001$ & 20 & 15 \\
\hline & $\mathrm{F}_{\mathrm{S3}}$ & 395 & $1.57(1.34-1.85)$ & $7.04(4.93-10.03)$ & $1.47(0.15)$ & $<0.001$ & 17 & 15 \\
\hline & $\mathrm{F}_{S 4}$ & 439 & $2.13(1.88-2.40)$ & $7.36(5.47-9.91)$ & $1.77(0.19)$ & $<0.001$ & 23 & 16 \\
\hline & $\mathrm{F}_{\mathrm{S5}}$ & 320 & $1.46(1.27-1.68)$ & $5.63(4.57-6.93)$ & $2.02(0.22)$ & $<0.001$ & 23 & 13 \\
\hline \multirow[t]{6}{*}{ Hunucma } & $\mathrm{F}_{\mathrm{SO}}$ & 355 & $0.34(0.28-0.40)$ & $1.42(1.03-1.96)$ & $1.16(0.09)$ & $<0.001$ & 3 & 3 \\
\hline & $\mathrm{F}_{\mathrm{S} 1}$ & 460 & $0.25(0.21-0.29)$ & $1.25(0.88-1.76)$ & $1.37(0.12)$ & $<0.001$ & 3 & 3 \\
\hline & $\mathrm{F}_{\mathrm{S2}}$ & 597 & $0.33(0.27-0.40)$ & $3.27(2.13-5.01)$ & $0.96(0.08)$ & $<0.001$ & 4 & 7 \\
\hline & $\mathrm{F}_{\mathrm{S3}}$ & 353 & $1.14(0.90-1.45)$ & $10.19(5.53-18.80)$ & $1.00(0.12)$ & $<0.001$ & 13 & 22 \\
\hline & $\mathrm{F}_{\mathrm{S4}}$ & 220 & $1.16(1.06-1.26)$ & $1.93(1.69-2.20)$ & $4.30(0.62)$ & $<0.001$ & 13 & 4 \\
\hline & $\mathrm{F}_{\mathrm{S5}}$ & 180 & $0.75(0.61-0.91)$ & $2.26(1.62-3.16)$ & $1.98(0.33)$ & $<0.001$ & 8 & 5 \\
\hline \multirow[t]{6}{*}{ Hoctun } & $\mathrm{F}_{\mathrm{SO}}$ & 535 & $0.48(0.41-0.56)$ & $1.85(1.48-2.32)$ & $1.63(0.14)$ & $<0.001$ & 5 & 4 \\
\hline & $\mathrm{F}_{\mathrm{S} 1}$ & 535 & $0.23(0.19-0.27)$ & $1.32(0.97-1.80)$ & $1.35(0.10)$ & $<0.001$ & 2 & 3 \\
\hline & $\mathrm{F}_{\mathrm{S2}}$ & 470 & $0.48(0.38-0.61)$ & $5.73(3.47-9.46)$ & $0.88(0.08)$ & $<0.001$ & 5 & 12 \\
\hline & $\mathrm{F}_{\mathrm{S} 3}$ & 508 & $0.57(0.48-0.67)$ & $2.92(2.13-3.99)$ & $1.34(0.12)$ & $<0.001$ & 6 & 6 \\
\hline & $\mathrm{F}_{S 4}$ & 380 & $0.75(0.65-0.86)$ & $2.61(1.92-3.56)$ & $1.76(0.23)$ & $<0.001$ & 8 & 6 \\
\hline & $\mathrm{F}_{\mathrm{S5}}$ & 440 & $0.94(0.79-1.12)$ & $4.19(3.09-5.66)$ & $1.47(0.14)$ & $<0.001$ & 10 & 9 \\
\hline \multirow[t]{6}{*}{ Agua Dulce } & $\mathrm{F}_{\mathrm{SO}}$ & 536 & $0.80(0.76-0.84)$ & $1.44(1.30-1.60)$ & $3.73(0.30)$ & $<0.001$ & 9 & 3 \\
\hline & $\mathrm{F}_{\mathrm{S} 1}$ & 480 & $1.06(1.01-1.12)$ & $1.80(1.62-2.01)$ & $4.15(0.38)$ & $<0.001$ & 12 & 4 \\
\hline & $\mathrm{F}_{\mathrm{S} 2}$ & 333 & $1.08(1.00-1.16)$ & $1.99(1.70-2.33)$ & $3.58(0.45)$ & $<0.001$ & 12 & 4 \\
\hline & $\mathrm{F}_{\mathrm{S3}}$ & 313 & $1.06(0.97-1.15)$ & $2.06(1.74-2.44)$ & $3.32(0.38)$ & $<0.001$ & 12 & 4 \\
\hline & $\mathrm{F}_{54}$ & 397 & $1.14(1.05-2.03)$ & $2.42(2.03-2.89)$ & $2.92(0.32)$ & $<0.001$ & 13 & 5 \\
\hline & $\mathrm{F}_{\mathrm{S5}}$ & 440 & $1.09(1.02-1.17)$ & $2.04(1.79-2.33)$ & $3.53(0.34)$ & $<0.001$ & 12 & 5 \\
\hline \multirow[t]{6}{*}{ Jose Cardel } & $\mathrm{F}_{\mathrm{SO}}$ & 420 & $1.26(1.21-1.34)$ & $2.18(1.95-2.48)$ & $4.01(1.18)$ & $<0.001$ & 14 & 5 \\
\hline & $\mathrm{F}_{\mathrm{S} 1}$ & 429 & $1.47(1.33-1.64)$ & $4.10(3.28-5.12)$ & $2.15(0.20)$ & $<0.001$ & 16 & 9 \\
\hline & $\mathrm{F}_{\mathrm{S2}}$ & 499 & $1.63(1.51-1.75)$ & $3.73(3.08-4.50)$ & $2.65(0.28)$ & $<0.001$ & 18 & 8 \\
\hline & $\mathrm{F}_{\mathrm{S3}}$ & 400 & $1.68(1.53-1.85)$ & $4.07(3.35-4.95)$ & $2.48(0.24)$ & $<0.001$ & 19 & 9 \\
\hline & $\mathrm{F}_{\mathrm{S4}}$ & 400 & $1.98(1.74-2.24)$ & $6.68(5.03-8.86)$ & $1.80(0.19)$ & $<0.001$ & 22 & 14 \\
\hline & $\mathrm{F}_{\mathrm{S5}}$ & 360 & $2.01(1.79-2.26)$ & $5.68(4.35-7.42)$ & $2.12(0.23)$ & $<0.001$ & 22 & 12 \\
\hline New Orleans (NO) & - & 492 & $0.09(0.08-0.11)$ & $0.46(0.32-0.65)$ & $1.43(0.13)$ & $<0.001$ & - & - \\
\hline
\end{tabular}

a sample size

b KC: $50 \%$ and $90 \%$ knockdown concentrations in micrograms per bottle, $95 \%$ confidence intervals

c RR: resistance ratio $\mathrm{KC}_{50}$ field strain/ $\mathrm{KC}_{50}$ susceptible strain

d b: logistic regression slope (standard error)

e New Orleans: susceptible reference strain

The values obtained after subtracting the initial reading (T0) from the reading at $10 \mathrm{~min}$ (T10) were used for the statistical analyses.
Total protein content was determined by the Bradford method [56], which was used to correct the activity values for all of the enzymes evaluated [57]. 
Table 3 Deltamethrin knockdown concentrations $\left(\mathrm{LC}_{50}\right.$ and $\mathrm{LC}_{90}$ ) and resistance ratios (RR) for Aedes aegypti females in the parental generation $\left(\mathrm{F}_{\mathrm{SO}}\right)$ and the deltamethrin-selected generations $\left(\mathrm{F}_{\mathrm{S} 1}-\mathrm{F}_{\mathrm{S} 5}\right)$

\begin{tabular}{|c|c|c|c|c|c|c|c|c|}
\hline \multirow[t]{2}{*}{ Population } & \multirow[t]{2}{*}{ Generation } & \multirow[t]{2}{*}{$\mathrm{n}^{\mathrm{a}}$} & \multicolumn{4}{|l|}{$L C^{b} \mu g / B$} & \multicolumn{2}{|l|}{$\mathrm{RR}^{\mathrm{c}}$} \\
\hline & & & $\mathrm{LC}_{50}(\mathrm{Cl})$ & $\mathrm{LC}_{90}(\mathrm{Cl})$ & $b^{d} \pm S E$ & $P$-value & $\overline{L C_{50}}$ & $\mathrm{LC}_{90}$ \\
\hline \multirow[t]{6}{*}{ Merida } & $\mathrm{F}_{\mathrm{SO}}$ & 466 & $5.35(4.73-6.56)$ & $42.11(27.77-63.84)$ & $1.06(0.09)$ & $<0.001$ & 134 & 140 \\
\hline & $\mathrm{F}_{\mathrm{S} 1}$ & 584 & $5.60(5.02-6.24)$ & $18.63(15.07-23.03)$ & $1.82(0.15)$ & $<0.001$ & 140 & 62 \\
\hline & $F_{S 2}$ & 525 & $5.80(5.16-6.49)$ & $19.32(19.96-30.27)$ & $1.82(0.18)$ & $<0.001$ & 145 & 64 \\
\hline & $\mathrm{F}_{\mathrm{S3}}$ & 520 & $7.87(7.03-8.82)$ & $24.58(19.96-30.27)$ & $1.93(0.17)$ & $<0.001$ & 196 & 82 \\
\hline & $\mathrm{F}_{\mathrm{S4}}$ & 501 & $7.73(6.93-8.61)$ & 24.15 (19.69-29.62) & $1.92(0.17)$ & $<0.001$ & 193 & 80 \\
\hline & $\mathrm{F}_{\mathrm{S5}}$ & 389 & $7.88(6.91-9.00)$ & $26.31(19.84-34.89)$ & $1.82(0.21)$ & $<0.001$ & 197 & 88 \\
\hline \multirow[t]{6}{*}{ Progreso } & $\mathrm{F}_{\mathrm{SO}}$ & 650 & $0.75(0.59-1.06)$ & $7.06(4.83-10.31)$ & $0.97(0.08)$ & $<0.001$ & 18 & 23 \\
\hline & $\mathrm{F}_{\mathrm{S} 1}$ & 473 & $1.06(0.86-1.29)$ & $7.72(5.21-11.44)$ & $1.10(0.09)$ & $<0.001$ & 26 & 26 \\
\hline & $\mathrm{F}_{\mathrm{S} 2}$ & 415 & $1.71(1.45-2.01)$ & $7.19(5.30-9.76)$ & $1.53(0.15)$ & $<0.001$ & 43 & 24 \\
\hline & $\mathrm{F}_{\mathrm{S3}}$ & 458 & $2.37(2.08-2.71)$ & $8.97(6.74-11.95)$ & $1.65(0.15)$ & $<0.001$ & 59 & 30 \\
\hline & $F_{S 4}$ & 461 & $2.06(1.86-2.28)$ & $5.63(4.57-6.93)$ & $2.18(0.20)$ & $<0.001$ & 51 & 19 \\
\hline & $\mathrm{F}_{\mathrm{S5}}$ & 320 & $1.66(1.46-1.88)$ & $4.32(3.23-5.78)$ & $2.02(0.22)$ & $<0.001$ & 36 & 14 \\
\hline \multirow[t]{6}{*}{ Hunucma } & $\mathrm{F}_{\mathrm{SO}}$ & 424 & $0.25(0.20-0.29)$ & $1.43(0.99-2.05)$ & $1.24(0.12)$ & $<0.001$ & 6 & 5 \\
\hline & $\mathrm{F}_{\mathrm{S} 1}$ & 359 & $0.69(0.53-0.78)$ & $3.50(2.32-5.28)$ & $1.30(0.14)$ & $<0.001$ & 17 & 12 \\
\hline & $\mathrm{F}_{\mathrm{S2}}$ & 497 & $0.80(0.69-0.93)$ & $3.63(2.68-4.91)$ & $1.45(0.14)$ & $<0.001$ & 20 & 12 \\
\hline & $\mathrm{F}_{\mathrm{S} 3}$ & 299 & $1.16(1.01-1.33)$ & $3.61(2.69-4.83)$ & $1.93(0.23)$ & $<0.001$ & 29 & 12 \\
\hline & $\mathrm{F}_{\mathrm{S4}}$ & 260 & $1.33(1.16-1.52)$ & $3.26(2.62-4.04)$ & $2.45(0.26)$ & $<0.001$ & 33 & 11 \\
\hline & $\mathrm{F}_{\mathrm{S5}}$ & 318 & $1.36(1.19-1.56)$ & $4.29(3.20-5.75)$ & $1.92(0.22)$ & $<0.001$ & 34 & 14 \\
\hline \multirow[t]{6}{*}{ Hoctun } & $\mathrm{F}_{\mathrm{SO}}$ & 532 & $1.30(1.15-1.46)$ & $4.06(3.25-5.08)$ & $1.92(0.16)$ & $<0.001$ & 32 & 13 \\
\hline & $F_{S 1}$ & 416 & $2.50(2.23-2.79)$ & $6.67(5.51-8.07)$ & $2.23(0.22)$ & $<0.001$ & 62 & 22 \\
\hline & $\mathrm{F}_{\mathrm{S} 2}$ & 467 & $3.27(3.01-3.55)$ & $7.43(6.28-8.78)$ & $2.67(0.27)$ & $<0.001$ & 82 & 23 \\
\hline & $\mathrm{F}_{\mathrm{S} 3}$ & 417 & $2.76(2.52-3.02)$ & $6.60(5.45-8.00)$ & $2.52(0.26)$ & $<0.001$ & 69 & 22 \\
\hline & $\mathrm{F}_{54}$ & 457 & $2.67(2.45-2.92)$ & $6.51(5.36-7.89)$ & $2.47(0.26)$ & $<0.001$ & 67 & 22 \\
\hline & $\mathrm{F}_{\mathrm{S5}}$ & 359 & $2.07(1.89-2.27)$ & $4.37(3.72-5.13)$ & $2.94(0.33)$ & $<0.001$ & 52 & 15 \\
\hline \multirow[t]{6}{*}{ Agua Dulce } & $\mathrm{F}_{\mathrm{SO}}$ & 475 & $1.00(0.91-1.09)$ & $2.62(2.14-3.21)$ & $2.27(0.21)$ & $<0.001$ & 25 & 7 \\
\hline & $F_{S 1}$ & 540 & $1.41(1.31-1.52)$ & $3.15(2.65-3.75)$ & $2.74(0.24)$ & $<0.001$ & 32 & 10 \\
\hline & $\mathrm{F}_{\mathrm{S} 2}$ & 420 & $2.02(1.81-2.25)$ & $5.51(4.49-6.78)$ & $2.18(0.21)$ & $<0.001$ & 50 & 18 \\
\hline & $\mathrm{F}_{\mathrm{S} 3}$ & 457 & $2.12(1.90-2.35)$ & $6.19(4.91-7.73)$ & $2.05(0.19)$ & $<0.001$ & 53 & 20 \\
\hline & $\mathrm{F}_{54}$ & 519 & $2.03(1.86-2.22)$ & $5.32(4.40-6.44)$ & $2.28(0.20)$ & $<0.001$ & 51 & 18 \\
\hline & $\mathrm{F}_{\mathrm{S5}}$ & 340 & $1.95(1.74-2.20)$ & $6.40(4.79-8.55)$ & $1.85(0.19)$ & $<0.001$ & 49 & 21 \\
\hline \multirow[t]{6}{*}{ Jose Cardel } & $\mathrm{F}_{\mathrm{SO}}$ & 481 & $1.64(1.46-1.83)$ & $5.22(4.10-6.64)$ & $1.89(0.18)$ & $<0.001$ & 41 & 6 \\
\hline & $\mathrm{F}_{\mathrm{S} 1}$ & 531 & $2.30(2.12-2.49)$ & $5.33(4.53-6.28)$ & $2.61(0.25)$ & $<0.001$ & 57 & 18 \\
\hline & $\mathrm{F}_{\mathrm{S2}}$ & 460 & $2.43(2.21-2.67)$ & $6.01(4.95-7.30)$ & $2.42(0.22)$ & $<0.001$ & 60 & 20 \\
\hline & $\mathrm{F}_{\mathrm{S3}}$ & 360 & $2.35(2.14-2.58)$ & $5.12(4.33-6.06)$ & $2.82(0.27)$ & $<0.001$ & 56 & 17 \\
\hline & $\mathrm{F}_{S 4}$ & 420 & $2.26(2.07-2.45)$ & $4.93(4.27-5.69)$ & $2.81(0.25)$ & $<0.001$ & 56 & 16 \\
\hline & $\mathrm{F}_{\mathrm{S5}}$ & 320 & $2.84(2.63-3.08)$ & $5.49(4.67-6.44)$ & $3.34(0.34)$ & $<0.001$ & 71 & 18 \\
\hline New Orleans (NO) & - & 492 & $0.04(0.03-0.05)$ & $0.30(0.21-0.45)$ & $1.11(0.10)$ & $<0.001$ & - & - \\
\hline
\end{tabular}

a sample size

b LC: $50 \%$ and $90 \%$ lethal concentrations in micrograms per bottle, $95 \%$ confidence intervals

c RR: resistance ratio $L C_{50}$ field strain/ $L_{50}$ susceptible strain

d $b$ : slope of the regression line log-Probit (standard error)

e New Orleans: susceptible reference strain

Positive and negative controls were included for MFOs and esterases. The same volume of homogenate which was used in the respective assays was used in running the controls. For the $\alpha$ - and $\beta$-esterases, $\alpha$ - and $\beta$-naphthyl acetate solution was used as positive controls, respectively. Cytochrome-C (Merck, Darmstadt, Germany) solution was the positive control for the MFO assay. 

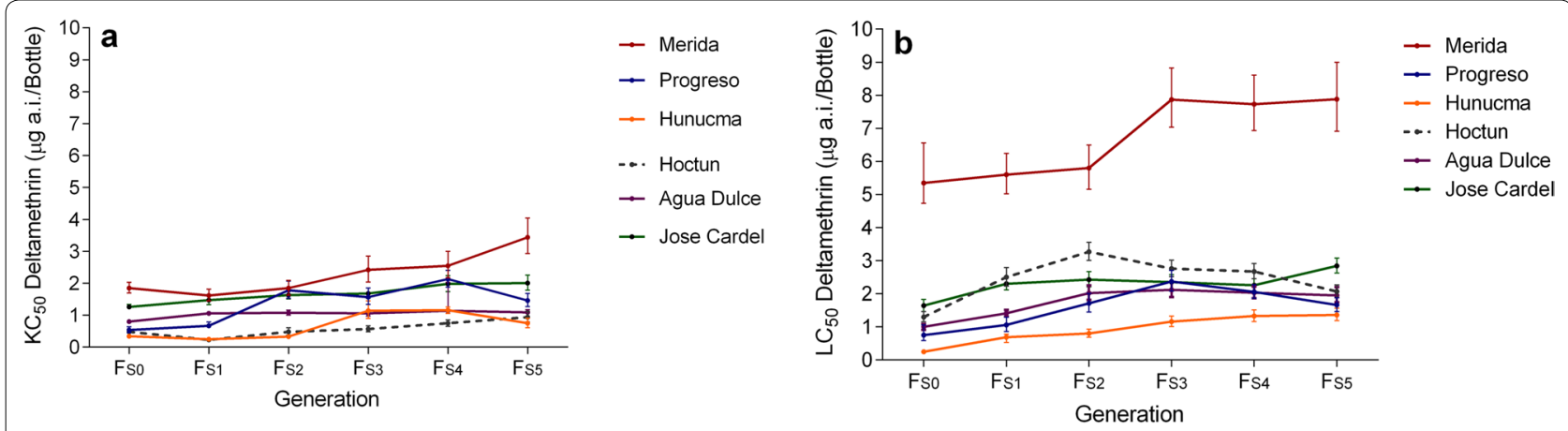

Fig. 2 a Knockdown concentrations values $\left(K C_{50}\right)$ as response to the selection with deltamethrin. $\mathbf{b}$ Lethal concentrations values $\left(L C_{50}\right)$ as response to the selection with deltamethrin in six populations of Aedes aegypti through five generations

$\mathrm{KPO}_{4}$ buffer was used as a negative control for each biochemical assay.

\section{Calibration curves}

The calibration curves were generated using control solutions for each enzyme family. $\alpha$ - or $\beta$-naphthol (SigmaAldrich) was used for $\alpha$-esterases and $\beta$-esterases, with a concentration range of 0.3 to $5 \mu \mathrm{g} / \mu \mathrm{l}$ and 0.5 to $4 \mu \mathrm{g} / \mu \mathrm{l}$, respectively. Cytochrome $\mathrm{C}$ concentrations from 0.002 to $0.65 \mu \mathrm{g} / \mu \mathrm{l}$ were used for MFOs, and 0.1 to $6.5 \mu \mathrm{g} / \mu \mathrm{l} \mathrm{BSA}$ solutions were used for total protein determination.

The absorbance values obtained for each enzyme were used to calculate the mean absorbance values per mosquito, which was then converted to enzyme activity after accounting for the homogenization volume, total protein content and the activity unit considered for each enzyme. Each value was multiplied by a conversion factor that was obtained from the calibration curves $[57,58]$.

The mean activity values underwent an analysis of variance (ANOVA, $P<0.05)$ and Tukey's multiple comparison of means $(P<0.05)$. Normality of the variance was verified by the Bartlett test. Statistical analyses were carried out with GraphPad Prism v.7 (GraphPad Software, Inc, Version 6.01, La Jolla, CA, USA; https://www.graph pad.com). Activity levels for all enzymes were determined in the parental population and in each deltamethrin-selected generation $\left(\mathrm{F}_{\mathrm{S} 0}-\mathrm{F}_{\mathrm{S} 5}\right)$.

The activity values corresponding to the 99th percentile of the New Orleans reference strain were calculated for each enzyme family. The average enzymatic activity was classified as non-altered (NA) when $<15 \%$ of individuals did not exceed the 99th percentile of the reference strain, incipiently altered (IA) if $15-50 \%$ of the individuals exceeded the 99th percentile, and altered (A) when this percentage exceeded $50 \%$, according to the criteria established by Montella et al. [59]. Additionally, the $\mathrm{LC}_{50}$ values and mean enzyme activity values of the parental generation and each selected generation $\left(\mathrm{F}_{\mathrm{S} 1}-\mathrm{F}_{\mathrm{S} 5}\right)$ underwent linear regression analysis. Correlation coefficients were calculated to determine the degree of association between the two variables.

\section{Results}

Bioassays

Deltamethrin $\mathrm{KC}_{50}$ and $\mathrm{LC}_{50}$ were determined for the parental generation and in each selected generation $\left(\mathrm{F}_{\mathrm{S} 1}-\mathrm{F}_{\mathrm{S} 5}\right)$ as well as for the insecticide-susceptible New Orleans (NO) reference strain. Although some variation was observed, $\mathrm{KC}_{50}$ and $\mathrm{LC}_{50}$ generally increased with respect to the parental generation $\left(\mathrm{F}_{\mathrm{S} 5} v s \mathrm{~F}_{\mathrm{S} 0}\right)$ (Tables 2, 3, Fig. 2).

When comparing the $\mathrm{LC}_{50}$ values obtained from the parental generations to the NO strain, five of the populations (Merida, Progreso, Hoctun, Agua Dulce and Jose Cardel) were highly resistant to deltamethrin with an $\mathrm{RR}_{\mathrm{LC50}}$ of 18-134-fold, and only one population (Hunucma) showed moderate resistance $\left(\mathrm{RR}_{\mathrm{LC} 50}\right.$ of 6-fold) (Table 3).

With respect to knockdown in the parental generation, $R_{K C 50}$ was high in two populations, Merida with $R_{K_{K C 50}}$ of 20 -fold and Jose Cardel with $\mathrm{RR}_{\mathrm{KC} 50}$ of 14-fold. Three populations (Hoctun, Progreso and Agua Dulce) showed moderate resistance with $\mathrm{RR}_{\mathrm{KC} 50}$ ranging between 5-9fold, and one population (Hunucma) had a low $\mathrm{RR}_{\mathrm{KC} 50}$ of 3-fold (Table 2).

When the populations were selected with deltamethrin, the increase in $\mathrm{LC}_{50}$ was not significant for $\mathrm{F}_{\mathrm{S} 1}$ and $\mathrm{F}_{\mathrm{S} 2}$ for the Merida population (based on overlapping 95\% CIs). However, there was a significant increase in $\mathrm{LC}_{50}$ from $\mathrm{F}_{\mathrm{S} 1}$ to $\mathrm{F}_{\mathrm{S} 5}$ in all other populations of Ae. aegypti (Table 3). For $\mathrm{KC}_{50}$, the increases were significant between all generations of selection $\left(\mathrm{F}_{\mathrm{S} 1}-\mathrm{F}_{\mathrm{S} 5}\right)$ for the Progreso, Agua Dulce and Jose Cardel populations but were not significant for 
Table 4 Estimation of heritability $\left(h^{2}\right)$ of resistance in Aedes aegypti after deltamethrin selection for knockdown concentration $\left(K_{50}\right)$

\begin{tabular}{|c|c|c|c|c|c|c|c|c|c|c|c|}
\hline Population & Generation & $\mathrm{KC}_{50}$ & $b$ & $\delta t$ & $\mathrm{Pt}$ & it & S & $\Sigma S$ & $\mathrm{R}$ & $\Sigma R$ & h2 \\
\hline \multirow[t]{6}{*}{ Merida } & $\mathrm{F}_{\mathrm{SO}}$ & 1.855 & 2.959 & 0.338 & - & - & - & - & - & - & \\
\hline & $\mathrm{F}_{\mathrm{S} 1}$ & 1.628 & 2.094 & 0.478 & 21.920 & 1.346 & 0.643 & 0.643 & & 0.000 & \\
\hline & $\mathrm{F}_{\mathrm{S2}}$ & 1.852 & 1.699 & 0.589 & 24.010 & 1.295 & 0.762 & 1.405 & 0.224 & 0.224 & \\
\hline & $\mathrm{F}_{\mathrm{S} 3}$ & 2.421 & 1.196 & 0.836 & 20.670 & 1.372 & 1.147 & 2.552 & 0.569 & 0.793 & \\
\hline & $\mathrm{F}_{54}$ & 2.556 & 1.364 & 0.733 & 24.030 & 1.295 & 0.949 & 3.502 & 0.135 & 0.928 & \\
\hline & $\mathrm{F}_{55}$ & 3.446 & 1.505 & 0.664 & - & - & & & 0.890 & 1.818 & 0.519 \\
\hline \multirow[t]{6}{*}{ Progreso } & $\mathrm{F}_{\mathrm{SO}}$ & 0.541 & 1.231 & 0.812 & - & - & - & - & - & - & \\
\hline & $\mathrm{F}_{\mathrm{S} 1}$ & 0.673 & 1.500 & 0.667 & 24.770 & 1.271 & 0.847 & 0.847 & & 0.000 & \\
\hline & $\mathrm{F}_{\mathrm{S2}}$ & 1.782 & 1.584 & 0.631 & 24.870 & 1.271 & 0.802 & 1.650 & 1.109 & 1.109 & \\
\hline & $\mathrm{F}_{\mathrm{S3}}$ & 1.579 & 1.470 & 0.680 & 24.480 & 1.295 & 0.881 & 2.531 & 0.203 & 1.312 & \\
\hline & $\mathrm{F}_{54}$ & 2.131 & 1.771 & 0.565 & 24.640 & 1.271 & 0.718 & 3.248 & 0.552 & 1.864 & \\
\hline & $\mathrm{F}_{\mathrm{S5}}$ & 1.465 & 2.028 & 0.493 & - & - & - & - & 0.666 & 2.530 & 0.779 \\
\hline \multirow[t]{6}{*}{ Hucnucma } & $\mathrm{F}_{\mathrm{SO}}$ & 0.343 & 1.169 & 0.856 & - & - & - & - & - & - & \\
\hline & $\mathrm{F}_{\mathrm{S} 1}$ & 0.253 & 1.376 & 0.727 & 24.730 & 1.271 & 0.924 & 0.924 & & 0.000 & \\
\hline & $\mathrm{F}_{\mathrm{S2}}$ & 0.334 & 0.964 & 1.037 & 24.670 & 1.271 & 1.318 & 2.242 & 0.081 & 0.081 & \\
\hline & $\mathrm{F}_{\mathrm{S3}}$ & 1.145 & 1.005 & 0.995 & 24.400 & 1.295 & 1.289 & 3.531 & 0.811 & 0.892 & \\
\hline & $\mathrm{F}_{54}$ & 1.160 & 4.308 & 0.232 & 24.480 & 1.295 & 0.301 & 3.831 & 0.015 & 0.907 & \\
\hline & $F_{55}$ & 0.751 & 1.987 & 0.503 & - & - & - & - & 0.409 & 1.316 & 0.343 \\
\hline \multirow[t]{6}{*}{ Hoctun } & $\mathrm{F}_{50}$ & 0.484 & 1.633 & 0.612 & - & - & - & - & - & - & \\
\hline & $\mathrm{F}_{\mathrm{S} 1}$ & 0.231 & 1.358 & 0.736 & 24.780 & 1.271 & 0.936 & 0.936 & & 0.000 & \\
\hline & $F_{S 2}$ & 0.484 & 0.889 & 1.125 & 24.620 & 1.271 & 1.430 & 2.366 & 0.253 & 0.253 & \\
\hline & $\mathrm{F}_{\mathrm{S} 3}$ & 0.573 & 1.348 & 0.742 & 24.460 & 1.295 & 0.961 & 3.326 & 0.089 & 0.342 & \\
\hline & $\mathrm{F}_{54}$ & 0.754 & 1.764 & 0.567 & 24.860 & 1.271 & 0.721 & 4.047 & 0.181 & 0.523 & \\
\hline & $\mathrm{F}_{\mathrm{S5}}$ & 0.949 & 1.479 & 0.676 & - & - & - & - & 0.195 & 0.718 & 0.177 \\
\hline \multirow[t]{6}{*}{ Agua Dulce } & $\mathrm{F}_{\mathrm{SO}}$ & 0.802 & 3.734 & 0.268 & - & - & - & - & - & - & \\
\hline & $F_{S 1}$ & 1.066 & 4.157 & 0.241 & 24.730 & 1.271 & 0.306 & 0.306 & & 0.000 & \\
\hline & $\mathrm{F}_{\mathrm{S2}}$ & 1.081 & 3.589 & 0.279 & 24.850 & 1.271 & 0.354 & 0.660 & 0.015 & 0.015 & \\
\hline & $\mathrm{F}_{\mathrm{S} 3}$ & 1.064 & 3.321 & 0.301 & 24.290 & 1.295 & 0.390 & 1.050 & 0.017 & 0.032 & \\
\hline & $\mathrm{F}_{\mathrm{S4}}$ & 1.146 & 2.929 & 0.341 & 24.540 & 1.271 & 0.434 & 1.484 & 0.082 & 0.114 & \\
\hline & $\mathrm{F}_{\mathrm{S5}}$ & 1.098 & 3.530 & 0.283 & - & - & - & - & 0.048 & 0.162 & 0.109 \\
\hline \multirow[t]{6}{*}{ Jose Cardel } & $\mathrm{F}_{50}$ & 1.267 & 4.018 & 0.249 & - & - & - & - & - & - & \\
\hline & $F_{S 1}$ & 1.478 & 2.152 & 0.465 & 24.210 & 1.295 & 0.602 & 0.602 & & 0.000 & \\
\hline & $\mathrm{F}_{\mathrm{S2}}$ & 1.631 & 2.655 & 0.377 & 24.370 & 1.295 & 0.488 & 1.090 & 0.153 & 0.153 & \\
\hline & $\mathrm{F}_{\mathrm{S3}}$ & 1.686 & 2.486 & 0.402 & 22.480 & 1.346 & 0.541 & 1.631 & 0.055 & 0.208 & \\
\hline & $\mathrm{F}_{54}$ & 1.981 & 1.808 & 0.553 & 24.130 & 1.295 & 0.716 & 2.347 & 0.295 & 0.503 & \\
\hline & $\mathrm{F}_{\mathrm{S5}}$ & 2.017 & 2.120 & 0.472 & - & - & - & - & 0.036 & 0.539 & 0.230 \\
\hline
\end{tabular}

the Merida and Hunucma populations for $\mathrm{F}_{\mathrm{S} 1}-\mathrm{F}_{\mathrm{S} 2}$ and the Hoctun population for $\mathrm{F}_{\mathrm{S} 1}-\mathrm{F}_{\mathrm{S} 3}$ (Table 2).

When comparing the $\mathrm{LC}_{50}$ and $\mathrm{KC}_{50}$ values for the last deltamethrin-selected generation $\left(\mathrm{F}_{\mathrm{S} 5}\right)$ vs the parental generation $\left(\mathrm{F}_{\mathrm{S} 0}\right)$, we observed an increase of $\sim 1.5-5.5$ times for $\mathrm{LC}_{50}$ and $\sim 1.4-2.7$ times for $\mathrm{KC}_{50}$ in all populations (Fig. 2).

The results revealed that deltamethrin resistance increased in response to selection pressure (Tables 2, 3, Fig. 2). The knockdown resistance ratio $\left(\mathrm{RR}_{\mathrm{KC} 50}\right)$ value increased from 20-fold to 38-fold from $\mathrm{F}_{\mathrm{S} 0}-\mathrm{F}_{\mathrm{S} 5}$ in the
Merida population, from 6-fold to 23-fold for Progreso, from 3-fold to 8-fold for Hunucma, from 5-fold to 10-fold in Hoctun, and slight increases were observed in the Agua Dulce and Jose Cardel populations with 9-12-fold and 14-22-fold differences, respectively. Lethal concentrations also increased between $\mathrm{F}_{\mathrm{S} 0}$ and $\mathrm{F}_{\mathrm{S} 5}$, with the $\mathrm{RR}_{\mathrm{LC50}}$ increasing in the Hunucma populations from 6-fold to 34-fold, doubling in the Agua Dulce and Progreso populations (25-49-fold and 18-36-fold, respectively) and with lower increases detected in the Merida, Hoctun and Jose Cardel populations (134-197-fold, 
Table 5 Estimation of heritability $\left(h^{2}\right)$ of resistance in Aedes aegypti after deltamethrin selection for lethal concentration $\left(L C_{50}\right)$

\begin{tabular}{|c|c|c|c|c|c|c|c|c|c|c|c|}
\hline Population & Generation & $\mathrm{LC}_{50}$ & $b$ & $\delta t$ & Pt & it & $S$ & $\Sigma S$ & $\mathrm{R}$ & $\Sigma \mathrm{R}$ & $h^{2}$ \\
\hline \multirow[t]{6}{*}{ Merida } & $\mathrm{F}_{\mathrm{SO}}$ & 5.350 & 1.066 & 0.938 & - & - & - & - & - & - & \\
\hline & $\mathrm{F}_{\mathrm{S} 1}$ & 5.600 & 1.827 & 0.547 & 22.130 & 1.346 & 0.737 & 0.737 & & 0.000 & \\
\hline & $\mathrm{F}_{\mathrm{S} 2}$ & 5.800 & 1.824 & 0.548 & 23.990 & 1.295 & 0.710 & 1.447 & 0.200 & 0.200 & \\
\hline & $\mathrm{F}_{\mathrm{S} 3}$ & 7.870 & 1.930 & 0.518 & 20.710 & 1.400 & 0.725 & 2.172 & 2.070 & 2.270 & \\
\hline & $\mathrm{F}_{54}$ & 7.730 & 1.926 & 0.519 & 24.120 & 1.295 & 0.672 & 2.844 & 0.140 & 2.410 & \\
\hline & $\mathrm{F}_{55}$ & 7.880 & 1.824 & 0.548 & - & - & - & - & 0.150 & 2.560 & 0.900 \\
\hline \multirow{6}{*}{ Progreso } & $\mathrm{F}_{50}$ & 0.749 & 0.979 & 1.021 & - & - & - & - & - & - & \\
\hline & $\mathrm{F}_{\mathrm{S} 1}$ & 1.060 & 1.107 & 0.904 & 24.79 & 1.271 & 1.149 & 1.149 & & 0.000 & \\
\hline & $\mathrm{F}_{\mathrm{S} 2}$ & 1.715 & 1.532 & 0.653 & 24.860 & 1.271 & 0.830 & 1.978 & 0.655 & 0.655 & \\
\hline & $\mathrm{F}_{\mathrm{S3}}$ & 2.378 & 1.654 & 0.605 & 24.460 & 1.295 & 0.783 & 2.761 & 0.663 & 1.318 & \\
\hline & $\mathrm{F}_{54}$ & 2.063 & 2.186 & 0.457 & 24.580 & 1.271 & 0.581 & 3.343 & 0.315 & 1.633 & \\
\hline & $\mathrm{F}_{\mathrm{S5}}$ & 1.663 & 2.008 & 0.498 & - & - & - & - & 0.400 & 2.033 & 0.608 \\
\hline \multirow[t]{6}{*}{ Hucnucma } & $\mathrm{F}_{\mathrm{SO}}$ & 0.245 & 1.244 & 0.804 & - & - & - & - & - & - & \\
\hline & $\mathrm{F}_{\mathrm{S1}}$ & 0.694 & 1.393 & 0.718 & 24.680 & 1.271 & 0.912 & 0.912 & & 0.000 & \\
\hline & $\mathrm{F}_{\mathrm{S2}}$ & 0.805 & 1.458 & 0.686 & 24.670 & 1.271 & 0.872 & 1.784 & 0.111 & 0.111 & \\
\hline & $\mathrm{F}_{\mathrm{S3}}$ & 1.164 & 1.940 & 0.516 & 24.240 & 1.295 & 0.668 & 2.452 & 0.359 & 0.470 & \\
\hline & $\mathrm{F}_{54}$ & 1.331 & 2.453 & 0.408 & 24.480 & 1.295 & 0.528 & 2.980 & 0.167 & 0.637 & \\
\hline & $\mathrm{F}_{55}$ & 1.370 & 1.922 & 0.520 & - & - & - & - & 0.039 & 0.676 & 0.227 \\
\hline \multirow[t]{6}{*}{ Hoctun } & $\mathrm{F}_{\mathrm{SO}}$ & 1.302 & 1.928 & 0.519 & - & - & - & - & - & - & \\
\hline & $\mathrm{F}_{\mathrm{S} 1}$ & 2.501 & 2.239 & 0.447 & 24.79 & 1.271 & 0.568 & 0.568 & & 0.000 & \\
\hline & $\mathrm{F}_{\mathrm{S2}}$ & 3.270 & 2.676 & 0.374 & 23.830 & 1.271 & 0.475 & 1.042 & 0.769 & 0.769 & \\
\hline & $\mathrm{F}_{\mathrm{S} 3}$ & 2.764 & 2.521 & 0.397 & 24.410 & 1.295 & 0.514 & 1.556 & 0.506 & 1.275 & \\
\hline & $\mathrm{F}_{S 4}$ & 2.677 & 2.472 & 0.405 & 24.890 & 1.271 & 0.514 & 2.070 & 0.087 & 1.362 & \\
\hline & $\mathrm{F}_{\mathrm{S5}}$ & 2.076 & 2.950 & 0.339 & - & - & - & - & 0.601 & 1.963 & 0.948 \\
\hline \multirow[t]{6}{*}{ Agua Dulce } & $\mathrm{F}_{\mathrm{SO}}$ & 1.000 & 2.278 & 0.439 & - & - & - & - & - & - & \\
\hline & $\mathrm{F}_{\mathrm{S} 1}$ & 1.410 & 2.741 & 0.365 & 24.77 & 1.271 & 0.464 & 0.464 & & 0.000 & \\
\hline & $\mathrm{F}_{\mathrm{S2}}$ & 2.023 & 2.190 & 0.457 & 24.820 & 1.271 & 0.580 & 1.044 & 0.613 & 0.613 & \\
\hline & $\mathrm{F}_{\mathrm{S} 3}$ & 2.120 & 2.058 & 0.486 & 24.280 & 1.295 & 0.629 & 1.674 & 0.097 & 0.710 & \\
\hline & $\mathrm{F}_{54}$ & 2.035 & 2.285 & 0.438 & 24.520 & 1.271 & 0.556 & 2.230 & 0.085 & 0.795 & \\
\hline & $\mathrm{F}_{\mathrm{S5}}$ & 1.957 & 1.855 & 0.539 & - & - & - & - & 0.078 & 0.873 & 0.392 \\
\hline \multirow[t]{6}{*}{ José Cardel } & $\mathrm{F}_{\mathrm{SO}}$ & 1.638 & 1.895 & 0.528 & - & - & - & - & - & - & \\
\hline & $F_{S 1}$ & 2.305 & 2.617 & 0.382 & 24.210 & 1.295 & 0.495 & 0.495 & & 0.000 & \\
\hline & $F_{S 2}$ & 2.436 & 2.429 & 0.412 & 24.370 & 1.295 & 0.533 & 1.028 & 0.131 & 0.131 & \\
\hline & $\mathrm{F}_{\mathrm{S3}}$ & 2.356 & 2.827 & 0.354 & 22.640 & 1.320 & 0.467 & 1.495 & 0.080 & 0.211 & \\
\hline & $\mathrm{F}_{54}$ & 2.260 & 2.814 & 0.355 & 24.130 & 1.295 & 0.460 & 1.955 & 0.096 & 0.307 & \\
\hline & $\mathrm{F}_{\mathrm{S5}}$ & 2.849 & 3.346 & 0.299 & - & - & - & - & 0.589 & 0.896 & 0.458 \\
\hline
\end{tabular}

32-52-fold and 41-71-fold, respectively). When analyzing the relative increases in $\mathrm{RR}_{\mathrm{KC} 50}$ and $\mathrm{RR}_{\mathrm{LC50}}$ during the selection, the Hunucma population showed the highest single-generation increase ( $>0.3$-fold) from $\mathrm{F}_{\mathrm{S} 1} v s \mathrm{~F}_{\mathrm{S} 2}$ for $\mathrm{KC}_{50}$; Hunucma showed also the highest increase in $\mathrm{RR}_{\mathrm{LC50}}$ (2.83) in the first selected generation relative to the parental generation $\left(\mathrm{F}_{\mathrm{S} 1} v s \mathrm{~F}_{\mathrm{S} 0}\right)$.

\section{Realized heritability}

The heritability of resistance $\left(\mathrm{h}^{2}\right)$ estimated over the generations of deltamethrin selection was highest in the
Hoctun and Merida populations, with values of 0.948 and 0.900 , respectively, for $\mathrm{LC}_{50}$. In the case of $\mathrm{KC}_{50}$, the highest values were obtained for the Progreso population (0.779) and Merida populations (0.519). The response to the selection ( $\mathrm{R}$ ) was also high for Merida and Progreso, for both $\mathrm{KC}_{50}$ and $\mathrm{LC}_{50}$. The selection differential (S) was high in the Progreso population for $\mathrm{LC}_{50}$, but in the case of $\mathrm{KC}_{50}$, the highest values were obtained for Hoctun and Hunucma. The number of generations required for a 10 -fold increase in $\mathrm{LC}_{50},(\mathrm{G})$, is the reciprocal of the response to selection (R) [47]. Thus, for the Progreso, 
Table 6 V410L genotypes and allele frequencies in Aedes aegypti females in the parental and all selected generations. $X^{2}$ HardyWeinberg, inbreeding coefficients $\left(F_{\mid S}\right)$ and significance testing was calculated for the parental generation

\begin{tabular}{|c|c|c|c|c|c|c|c|c|c|}
\hline Population & Generation & $n$ & $\mathrm{WV}$ & $\mathrm{VL}$ & $\mathrm{LL}$ & Freq. $(95 \% \mathrm{Cl})$ & $F_{1 S}$ & $\begin{array}{l}x^{2} \text { Hardy- } \\
\text { Weinberg }\end{array}$ & $P$-value \\
\hline \multirow[t]{6}{*}{ Merida } & $\mathrm{F}_{\mathrm{SO}}$ & 32 & 3 & 8 & 21 & $0.78(0.60-0.89)$ & 0.26 & 2.3 & 0.128 \\
\hline & $\mathrm{F}_{\mathrm{S} 1}$ & 29 & 5 & 14 & 10 & $0.59(0.40-0.74)$ & - & - & \\
\hline & $\mathrm{F}_{\mathrm{S} 2}$ & 32 & 4 & 11 & 17 & $0.70(0.52-0.83)$ & - & - & \\
\hline & $\mathrm{F}_{\mathrm{S3}}$ & 29 & 0 & 5 & 24 & $0.91(0.74-0.98)$ & - & - & \\
\hline & $\mathrm{F}_{S 4}$ & 32 & 1 & 8 & 23 & $0.84(0.67-0.93)$ & - & - & \\
\hline & $\mathrm{F}_{\mathrm{S5}}$ & 32 & 0 & 5 & 27 & $0.92(0.76-0.98)$ & - & - & \\
\hline \multirow[t]{6}{*}{ Progreso } & $\mathrm{F}_{\mathrm{SO}}$ & 30 & 4 & 13 & 13 & $0.65(0.47-0.79)$ & 0.04 & 0.06 & 0.794 \\
\hline & $\mathrm{F}_{\mathrm{S} 1}$ & 29 & 0 & 5 & 24 & $0.91(0.75-0.98)$ & - & - & \\
\hline & $\mathrm{F}_{\mathrm{S} 2}$ & 30 & 1 & 5 & 24 & $0.88(0.71-0.96)$ & - & - & \\
\hline & $\mathrm{F}_{\mathrm{S} 3}$ & 32 & 1 & 2 & 29 & $0.94(0.78-0.99)$ & - & - & \\
\hline & $\mathrm{F}_{\mathrm{S4}}$ & 30 & 1 & 12 & 17 & $0.77(0.58-0.88)$ & - & - & \\
\hline & $\mathrm{F}_{\mathrm{S5}}$ & 32 & 0 & 5 & 27 & $0.92(0.76-0.98)$ & - & - & \\
\hline \multirow[t]{6}{*}{ Hunucma } & $\mathrm{F}_{\mathrm{SO}}$ & 32 & 14 & 13 & 5 & $0.36(0.21-0.53)$ & 0.11 & 0.44 & 0.505 \\
\hline & $\mathrm{F}_{\mathrm{S} 1}$ & 32 & 7 & 13 & 12 & $0.58(0.40-0.73)$ & - & - & \\
\hline & $\mathrm{F}_{\mathrm{S} 2}$ & 21 & 6 & 9 & 6 & $0.50(0.30-0.69)$ & - & - & \\
\hline & $\mathrm{F}_{\mathrm{S3}}$ & 30 & 2 & 14 & 14 & $0.70(0.51-0.83)$ & - & - & \\
\hline & $\mathrm{F}_{S 4}$ & 32 & 1 & 3 & 28 & $0.92(0.76-0.98)$ & - & - & \\
\hline & $\mathrm{F}_{55}$ & 30 & 0 & 3 & 27 & $0.95(0.79-0.99)$ & - & - & \\
\hline \multirow[t]{6}{*}{ Hoctun } & $\mathrm{F}_{\mathrm{SO}}$ & 32 & 6 & 21 & 5 & $0.48(0.32-0.64)$ & -0.31 & 3.15 & 0.075 \\
\hline & $\mathrm{F}_{\mathrm{S} 1}$ & 31 & 15 & 11 & 5 & $0.34(0.19-0.51)$ & - & - & \\
\hline & $\mathrm{F}_{\mathrm{S} 2}$ & 28 & 4 & 13 & 11 & $0.63(0.44-0.77)$ & - & - & \\
\hline & $\mathrm{F}_{\mathrm{S} 3}$ & 31 & 0 & 4 & 27 & $0.94(0.78-0.99)$ & - & - & \\
\hline & $\mathrm{F}_{54}$ & 32 & 0 & 9 & 23 & $0.86(0.69-0.94)$ & - & - & \\
\hline & $\mathrm{F}_{\mathrm{S5}}$ & 30 & 0 & 8 & 22 & $0.87(0.69-0.95)$ & - & - & \\
\hline \multirow[t]{6}{*}{ Agua Dulce } & $\mathrm{F}_{\mathrm{SO}}$ & 30 & 6 & 15 & 9 & $0.55(0.37-0.71)$ & -0.01 & 0.003 & 0.956 \\
\hline & $\mathrm{F}_{\mathrm{S} 1}$ & 30 & 3 & 13 & 14 & $0.68(0.50-0.82)$ & - & - & \\
\hline & $\mathrm{F}_{\mathrm{S} 2}$ & 27 & 8 & 14 & 5 & $0.44(0.27-0.62)$ & - & - & \\
\hline & $\mathrm{F}_{\mathrm{S} 3}$ & 34 & 6 & 22 & 6 & $0.50(0.34-0.65)$ & - & - & \\
\hline & $\mathrm{F}_{\mathrm{S} 4}$ & 32 & 0 & 5 & 27 & $0.92(0.76-0.98)$ & - & - & \\
\hline & $\mathrm{F}_{\mathrm{S5}}$ & 32 & 0 & 1 & 31 & $0.98(0.84-1.00)$ & - & - & \\
\hline \multirow[t]{6}{*}{ Jose Cardel } & $\mathrm{F}_{\mathrm{SO}}$ & 32 & 4 & 14 & 14 & $0.66(0.48-0.79)$ & 0.03 & 0.02 & 0.863 \\
\hline & $\mathrm{F}_{\mathrm{S} 1}$ & 32 & 3 & 11 & 18 & $0.73(0.55-0.85)$ & - & - & \\
\hline & $\mathrm{F}_{\mathrm{S} 2}$ & 32 & 0 & 7 & 25 & $0.89(0.72-0.96)$ & - & - & \\
\hline & $\mathrm{F}_{\mathrm{S3}}$ & 30 & 0 & 7 & 23 & $0.88(0.71-0.96)$ & - & - & \\
\hline & $\mathrm{F}_{\mathrm{S4}}$ & 32 & 0 & 4 & 28 & $0.94(0.78-0.99)$ & - & - & \\
\hline & $\mathrm{F}_{\mathrm{S5}}$ & 30 & 1 & 10 & 19 & $0.80(0.62-0.90)$ & - & - & \\
\hline
\end{tabular}

Abbreviations: $\mathrm{n}$, sample size; V410/V410, wild type; V410/L410, heterozygotes; L410/L410, homozygotes resistant

Merida and Hoctun populations, an estimated mean of $\sim 2$ generations was necessary for a 10 -fold increase in $\mathrm{LC}_{50}$; however, for Hunucma, Agua Dulce and Jose Cardel, a mean of $>4$ generations was necessary. Regarding $\mathrm{KC}_{50}$, an estimated mean of $\sim 2$ generations was necessary for a 10-fold increase for the Merida and Progreso populations, but for Hunucma, Hoctun, Jose Cardel and Agua Dulce, means of 3, 6, 7 and $\sim 25$ generations, respectively, were necessary for a 10 -fold increase (Tables 4,5 ).

\section{Molecular assays}

Tables 6, 7, 8 summarize the frequency of L410, I1016 and $\mathrm{C} 1534$ alleles across all populations and generations $\left(\mathrm{F}_{\mathrm{S} 0}-\mathrm{F}_{\mathrm{S} 5}\right)$. The three $k d r$ mutations were present in all the parental populations, with frequencies ranging between $0.36-078$ for the L410 allele and $0.34-0.77$ for I1016. The highest frequencies were for C1534, ranging from 0.59-1. All populations were in Hardy-Weinberg equilibrium at the parental generation $\left(\mathrm{F}_{\mathrm{S} 0}\right)$, with the exception of Jose Cardel for the mutation F1534C. 
Table 7 V1016l genotypes and allele frequencies in Aedes aegypti females in the parental and all selected generations. $X^{2}$ HardyWeinberg, inbreeding coefficients $\left(F_{\mid S}\right)$ and significance testing was calculated for the parental generation

\begin{tabular}{|c|c|c|c|c|c|c|c|c|c|}
\hline Population & Generation & $n$ & W & $\mathrm{Vl}$ & $\|$ & Freq. (95\% Cl) & $F_{I S}$ & $\begin{array}{l}x^{2} \text { Hardy- } \\
\text { Weinberg }\end{array}$ & $P$-value \\
\hline \multirow[t]{6}{*}{ Merida } & $\mathrm{F}_{\mathrm{SO}}$ & 32 & 3 & 9 & 20 & $0.77(0.59-0.88)$ & 0.21 & 1.49 & 0.221 \\
\hline & $\mathrm{F}_{\mathrm{S} 1}$ & 30 & 5 & 15 & 10 & $0.58(0.40-0.73)$ & - & - & \\
\hline & $\mathrm{F}_{\mathrm{S} 2}$ & 32 & 4 & 10 & 18 & $0.72(0.54-0.84)$ & - & - & \\
\hline & $\mathrm{F}_{\mathrm{S} 3}$ & 31 & 0 & 7 & 24 & $0.89(0.72-0.96)$ & - & - & \\
\hline & $\mathrm{F}_{\mathrm{S4}}$ & 32 & 0 & 9 & 23 & $0.86(0.69-0.94)$ & - & - & \\
\hline & $\mathrm{F}_{\mathrm{S5}}$ & 32 & 0 & 5 & 27 & $0.92(0.76-0.98)$ & - & - & \\
\hline \multirow[t]{6}{*}{ Progreso } & $\mathrm{F}_{\mathrm{SO}}$ & 32 & 4 & 12 & 16 & $0.69(0.51-0.82)$ & 0.12 & 0.51 & 0.472 \\
\hline & $\mathrm{F}_{\mathrm{S} 1}$ & 32 & 0 & 22 & 10 & $0.66(0.48-0.79)$ & - & - & \\
\hline & $\mathrm{F}_{\mathrm{S2}}$ & 30 & 1 & 5 & 24 & $0.88(0.71-0.96)$ & - & - & \\
\hline & $\mathrm{F}_{\mathrm{S} 3}$ & 32 & 1 & 3 & 28 & $0.92(0.76-0.98)$ & - & - & \\
\hline & $\mathrm{F}_{S 4}$ & 32 & 1 & 12 & 19 & $0.78(0.60-0.89)$ & - & - & \\
\hline & $\mathrm{F}_{\mathrm{S5}}$ & 31 & 0 & 5 & 26 & $0.92(0.76-0.98)$ & - & - & \\
\hline \multirow[t]{6}{*}{ Hunucma } & $\mathrm{F}_{\mathrm{SO}}$ & 32 & 14 & 14 & 4 & $0.34(0.29-0.51)$ & 0.03 & 0.02 & 0.863 \\
\hline & $\mathrm{F}_{\mathrm{S} 1}$ & 32 & 7 & 14 & 11 & $0.56(0.39-0.71)$ & - & - & \\
\hline & $\mathrm{F}_{\mathrm{S} 2}$ & 32 & 7 & 19 & 6 & $0.48(0.32-0.64)$ & - & - & \\
\hline & $\mathrm{F}_{\mathrm{S3}}$ & 29 & 2 & 15 & 12 & $0.67(0.48-0.81)$ & - & - & \\
\hline & $\mathrm{F}_{\mathrm{S4}}$ & 32 & 1 & 2 & 29 & $0.94(0.78-0.99)$ & - & - & \\
\hline & $\mathrm{F}_{\mathrm{S5}}$ & 31 & 0 & 3 & 28 & $0.95(0.80-0.99)$ & - & - & \\
\hline \multirow[t]{6}{*}{ Hoctun } & $\mathrm{F}_{\mathrm{SO}}$ & 32 & 7 & 21 & 4 & $0.45(0.29-0.62)$ & -0.32 & 3.36 & 0.066 \\
\hline & $\mathrm{F}_{\mathrm{S} 1}$ & 32 & 16 & 11 & 5 & $0.33(0.19-0.50)$ & - & - & \\
\hline & $\mathrm{F}_{\mathrm{S} 2}$ & 27 & 4 & 14 & 9 & $0.59(0.40-0.75)$ & - & - & \\
\hline & $\mathrm{F}_{\mathrm{S3}}$ & 30 & 0 & 5 & 25 & $0.92(0.75-0.98)$ & - & - & \\
\hline & $\mathrm{F}_{\mathrm{S4}}$ & 32 & 0 & 10 & 22 & $0.84(0.67-0.93)$ & - & - & \\
\hline & $\mathrm{F}_{\mathrm{S5}}$ & 32 & 0 & 8 & 24 & $0.88(0.71-0.95)$ & - & - & \\
\hline \multirow[t]{6}{*}{ Agua Dulce } & $\mathrm{F}_{\mathrm{SO}}$ & 32 & 6 & 16 & 10 & $0.56(0.39-0.71)$ & -0.01 & 0.01 & 0.928 \\
\hline & $\mathrm{F}_{\mathrm{S} 1}$ & 32 & 4 & 14 & 14 & $0.66(0.48-0.79)$ & - & - & \\
\hline & $\mathrm{F}_{\mathrm{S2}}$ & 31 & 8 & 18 & 5 & $0.45(0.29-0.62)$ & - & - & \\
\hline & $\mathrm{F}_{\mathrm{S} 3}$ & 34 & 2 & 21 & 11 & $0.63(0.46-0.77)$ & - & - & \\
\hline & $\mathrm{F}_{\mathrm{S4}}$ & 32 & 0 & 4 & 28 & $0.94(0.78-0.99)$ & - & - & \\
\hline & $\mathrm{F}_{\mathrm{S5}}$ & 32 & 0 & 2 & 30 & $0.96(0.82-1.00)$ & - & - & \\
\hline \multirow[t]{6}{*}{ Jose Cardel } & $\mathrm{F}_{\mathrm{SO}}$ & 31 & 5 & 13 & 13 & $0.63(0.45-0.77)$ & 0.1 & 0.31 & 0.572 \\
\hline & $F_{S 1}$ & 30 & 7 & 8 & 15 & $0.63(0.45-0.78)$ & - & - & \\
\hline & $\mathrm{F}_{\mathrm{S} 2}$ & 32 & 0 & 8 & 24 & $0.88(0.71-0.95)$ & - & - & \\
\hline & $\mathrm{F}_{\mathrm{S} 3}$ & 30 & 0 & 17 & 13 & $0.72(0.53-0.84)$ & - & - & \\
\hline & $\mathrm{F}_{S 4}$ & 32 & 0 & 3 & 29 & $0.95(0.80-0.99)$ & - & - & \\
\hline & $\mathrm{F}_{\mathrm{S5}}$ & 31 & 0 & 5 & 26 & $0.92(0.76-0.98)$ & - & - & \\
\hline
\end{tabular}

Abbreviations: $\mathrm{n}$, sample size; V1016/V1016, wild type; V1016/11016, heterozygotes; |1016/11016, homozygotes resistant

A total of 20 combinations of tri-locus genotypes were detected among the populations in the parental generation $\left(\mathrm{F}_{\mathrm{S} 0}\right)$. Figure 3 shows the frequency of each of the 20 tri-locus genotype combinations. Triple homozygote resistant genotype $\left(\mathrm{LL}_{410} / \mathrm{II}_{1016} / \mathrm{CC}_{1534}\right)$ occurred in all parental $\left(\mathrm{F}_{\mathrm{S} 0}\right)$ populations of Ae. aegypti with the highest frequency for the Merida population (0.63) and the lowest for Hoctun (0.06), and their frequency increased as generations were subsequently selected (Fig. 4).
Interestingly, the triple heterozygous genotype $\left(\mathrm{VL}_{410} /\right.$ $\mathrm{VI}_{1016} / \mathrm{FC}_{1534}$ ) was only found in the populations of Hunucma, Hoctu and Jose Cardel, in which the triple wild-type genotype $\left(\mathrm{VV}_{410} / \mathrm{VI}_{1016} / \mathrm{FC}_{1534}\right)$ was also found.

In general, the frequencies of the three $k d r$ alleles increased over the selected generations. The frequencies of L410 from the parental generation to the last selected generation increased from 0.78 to 0.92 in the Merida population, 0.65 to 0.92 in Progreso, 0.36 to 0.95 
Table 8 F1534C genotypes and allele frequencies in Aedes aegypti females in the parental and all selected generations. $X^{2}$ HardyWeinberg, inbreeding coefficients $\left(F_{\mid S}\right)$ and significance testing was calculated for the parental generation

\begin{tabular}{|c|c|c|c|c|c|c|c|c|c|}
\hline Population & Generation & $\mathrm{n}$ & $\mathrm{FF}$ & FC & CC & Freq. $(95 \% \mathrm{Cl})$ & $F_{15}$ & $\begin{array}{l}x^{2} \text { Hardy- } \\
\text { Weinberg }\end{array}$ & $P$-value \\
\hline \multirow[t]{6}{*}{ Merida } & $\mathrm{F}_{\mathrm{SO}}$ & 32 & 0 & 0 & 32 & $1.00(0.86-1.00)$ & - & - & - \\
\hline & $\mathrm{F}_{\mathrm{S} 1}$ & 32 & 0 & 0 & 32 & $1.00(0.86-1.00)$ & - & - & \\
\hline & $\mathrm{F}_{\mathrm{S} 2}$ & 32 & 0 & 0 & 32 & $1.00(0.86-1.00)$ & - & - & \\
\hline & $\mathrm{F}_{\mathrm{S3}}$ & 31 & 0 & 0 & 31 & $1.00(0.86-1.00)$ & - & - & \\
\hline & $\mathrm{F}_{54}$ & 32 & 0 & 0 & 32 & $1.00(0.86-1.00)$ & - & - & \\
\hline & $\mathrm{F}_{\mathrm{S5}}$ & 32 & 0 & 0 & 32 & $1.00(0.86-1.00)$ & - & - & \\
\hline \multirow[t]{6}{*}{ Progreso } & $\mathrm{F}_{\mathrm{SO}}$ & 32 & 0 & 2 & 30 & $0.97(0.82-1.00)$ & -0.03 & 0.03 & 0.855 \\
\hline & $\mathrm{F}_{\mathrm{S} 1}$ & 32 & 0 & 0 & 32 & $1.00(0.86-1.00)$ & - & - & \\
\hline & $\mathrm{F}_{\mathrm{S} 2}$ & 30 & 0 & 0 & 30 & $1.00(0.86-1.00)$ & - & - & \\
\hline & $\mathrm{F}_{\mathrm{S3}}$ & 32 & 0 & 0 & 32 & $1.00(0.86-1.00)$ & - & - & \\
\hline & $\mathrm{F}_{\mathrm{S4}}$ & 32 & 0 & 0 & 32 & $1.00(0.86-1.00)$ & - & - & \\
\hline & $\mathrm{F}_{55}$ & 32 & 0 & 0 & 32 & $1.00(0.86-1.00)$ & - & - & \\
\hline \multirow[t]{6}{*}{ Hunucma } & $\mathrm{F}_{\mathrm{SO}}$ & 32 & 5 & 13 & 14 & $0.64(0.46-0.78)$ & 0.11 & 0.44 & 0.505 \\
\hline & $F_{S 1}$ & 32 & 2 & 6 & 24 & $0.84(0.67-0.93)$ & - & - & \\
\hline & $\mathrm{F}_{\mathrm{S} 2}$ & 32 & 5 & 4 & 23 & $0.78(0.60-0.89)$ & - & - & \\
\hline & $\mathrm{F}_{\mathrm{S} 3}$ & 29 & 2 & 7 & 20 & $0.81(0.62-0.91)$ & - & - & \\
\hline & $\mathrm{F}_{54}$ & 32 & 0 & 0 & 32 & $1.00(0.86-1.00)$ & - & - & \\
\hline & $\mathrm{F}_{\mathrm{S5}}$ & 32 & 0 & 0 & 32 & $1.00(0.86-1.00)$ & - & - & \\
\hline \multirow[t]{6}{*}{ Hoctun } & $\mathrm{F}_{\mathrm{sO}}$ & 32 & 4 & 18 & 10 & $0.59(0.42-0.74)$ & 0.16 & 0.88 & 0.347 \\
\hline & $\mathrm{F}_{\mathrm{S} 1}$ & 32 & 7 & 11 & 14 & $0.61(0.43-0.75)$ & - & - & \\
\hline & $\mathrm{F}_{\mathrm{S} 2}$ & 29 & 3 & 5 & 21 & $0.81(0.62-0.91)$ & - & - & \\
\hline & $\mathrm{F}_{\mathrm{S3}}$ & 30 & 0 & 0 & 30 & $1.00(0.86-1.00)$ & - & - & \\
\hline & $\mathrm{F}_{54}$ & 32 & 0 & 0 & 32 & $1.00(0.86-1.00)$ & - & - & \\
\hline & $\mathrm{F}_{\mathrm{S5}}$ & 32 & 0 & 0 & 32 & $1.00(0.86-1.00)$ & - & - & \\
\hline \multirow[t]{6}{*}{ Agua Dulce } & $\mathrm{F}_{\mathrm{SO}}$ & 32 & 0 & 0 & 32 & $1.00(0.86-1.00)$ & - & - & - \\
\hline & $\mathrm{F}_{\mathrm{S} 1}$ & 32 & 0 & 0 & 32 & $1.00(0.86-1.00)$ & - & - & \\
\hline & $\mathrm{F}_{\mathrm{S} 2}$ & 31 & 0 & 0 & 31 & $1.00(0.86-1.00)$ & - & - & \\
\hline & $\mathrm{F}_{\mathrm{S} 3}$ & 34 & 0 & 0 & 34 & $1.00(0.86-1.00)$ & - & - & \\
\hline & $\mathrm{F}_{\mathrm{S4}}$ & 32 & 0 & 0 & 32 & $1.00(0.86-1.00)$ & - & - & \\
\hline & $\mathrm{F}_{\mathrm{S5}}$ & 32 & 0 & 0 & 32 & $1.00(0.86-1.00)$ & - & - & \\
\hline \multirow[t]{6}{*}{ Jose Cardel } & $\mathrm{F}_{\mathrm{SO}}$ & 31 & 2 & 3 & 26 & $0.89(0.72-0.96)$ & 0.51 & 8.28 & 0.004 \\
\hline & $\mathrm{F}_{\mathrm{S} 1}$ & 32 & 0 & 0 & 32 & $1.00(0.86-1.00)$ & - & - & \\
\hline & $\mathrm{F}_{\mathrm{S} 2}$ & 32 & 0 & 0 & 32 & $1.00(0.86-1.00)$ & - & - & \\
\hline & $\mathrm{F}_{\mathrm{S} 3}$ & 30 & 0 & 0 & 30 & $1.00(0.86-1.00)$ & - & - & \\
\hline & $\mathrm{F}_{54}$ & 32 & 0 & 0 & 32 & $1.00(0.86-1.00)$ & - & - & \\
\hline & $\mathrm{F}_{55}$ & 32 & 0 & 0 & 32 & $1.00(0.86-1.00)$ & - & - & \\
\hline
\end{tabular}

Abbreviations: n, sample size; F1534/F1534, wild type; F1534/C1534, heterozygotes; C1534/C1534, homozygotes resistant

in Hunucma, 0.48 to 0.87 in Hoctun, 0.55 to 0.98 in Agua Dulce and 0.66 to 0.80 in Jose Cardel.

For the I1016 allele, increases of 0.77-0.92 were observed in the Merida population, 0.69-0.92 in Progreso, 0.45-0.95 in Hunucma, 0.34-0.88 in Hoctun, 0.56-0.96 in Agua Dulce and 0.63-0.92 in Jose Cardel.

The frequencies of the C1534 allele increased from 0.59 to 1 in the Hoctun population, 0.64 to 1 in
Hunucma, 0.89 to 1 in Jose Cardel and 0.97 to 1 in Progreso; the $\mathrm{C} 1534$ allele was fixed at 1.0 in the Merida and Agua Dulce populations in all generations. This allele reached fixation after the first selection with deltamethrin $\left(\mathrm{F}_{\mathrm{S} 1}\right)$ in the population from Progreso and Jose Cardel, in the third selected generation for Hoctun and in the fourth selected generation for Hunucma.

The L410 and I1016 alleles were selected in almost the same proportion from $\mathrm{F}_{\mathrm{S} 0}$ to the $\mathrm{F}_{\mathrm{S} 5}$ in all populations 


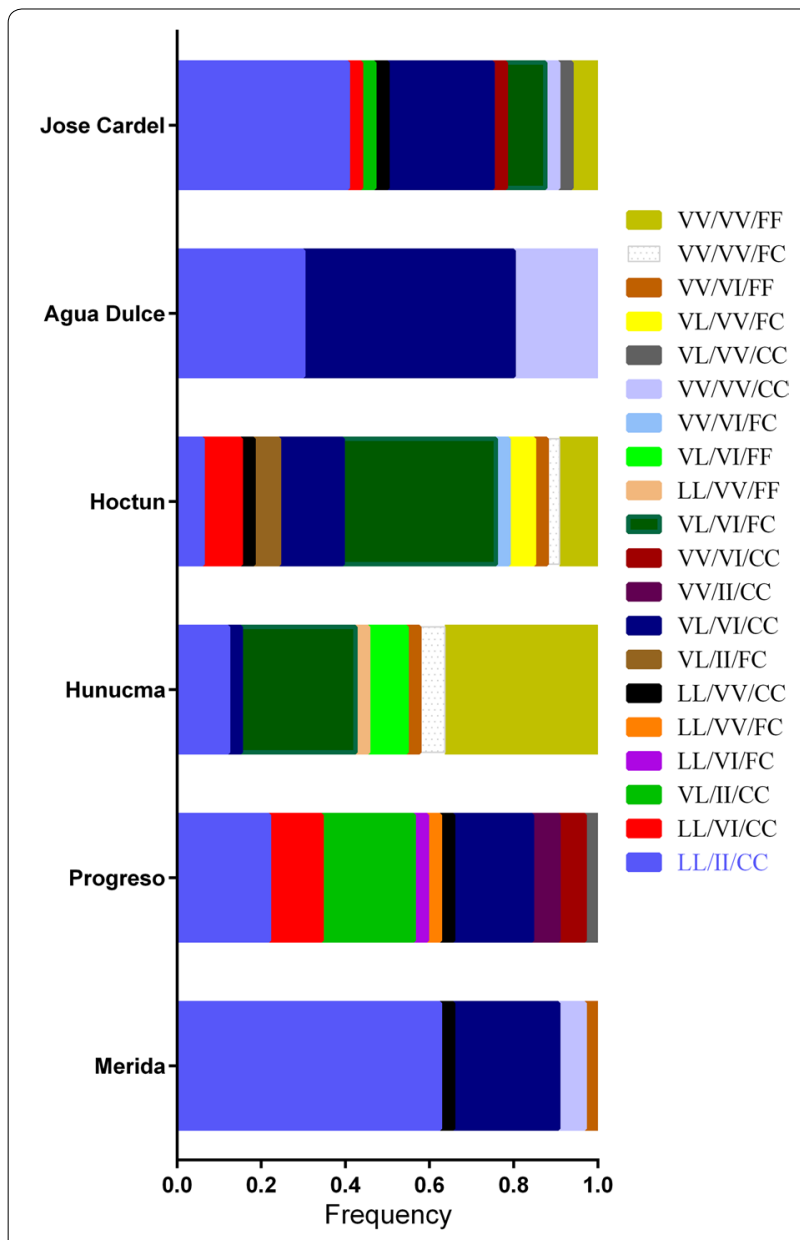

Fig. 3 Frequencies of the tri-locus genotypes in the parental generation $\left(F_{s 0}\right)$ of each population of Aedes aegypti. Genotypes order: 410/1016/1534

analyzed (Tables 6, 7, Fig. 5). Figure 6 shows that the increase in the frequencies of the L410 alleles in response to the selection with deltamethrin correlates significantly $(P<0.05)$ with the increase in the frequencies of the I106 allele by $98 \%$ in the population of Merida, 96\% in Hunucma, 95\% in Hoctun and 95\% in Agua Dulce. Additionally, in the populations of Hoctun and Hunucma we found that the allelic frequency of $\mathrm{C} 1534$ increased significantly $(P<0.05)$ with the increase in allele frequency of I1016 and L410 throughout the selection in the populations of Hunucma and Hoctun (Fig. 7).

The frequency of the mutant alleles for each mutation was correlated with the $\mathrm{KC}_{50}$ and $\mathrm{LC}_{50}$ values across the generations of selection (Table 9 ). $\mathrm{KC}_{50}$ values were significantly correlated with L410 frequencies in the Merida and Hoctun populations $(P<0.05)$; and with the frequencies of I1016 in the Merida, Hoctun and Jose Cardel $(P<0.05)$. Meanwhile, the frequencies of L410 and I1016 were significantly correlated with $\mathrm{LC}_{50}$ values in the Merida and Hunucma populations as well as $\mathrm{C} 1534$ correlated with $\mathrm{LC}_{50}$ values in Hunucma and Jose Cardel $(P<0.05)$. The presence and frequency of triple homozygous mutant individuals showed a significant correlation with $\mathrm{KC}_{50}$ only in the Merida population and $\mathrm{LC}_{50}$ in the Hunucma population $(P<0.05)$.

\section{Biochemical assays}

Table 10 shows the mean enzyme activity levels detected in the biochemical assays in the parental generation and all deltamethrin-selected generations, where at least 30 individuals per population were analyzed. When comparing values from the parental generations with the 99th percentile of the New Orleans reference strain, five of the six populations analyzed (Merida, Progreso, Hunucma, Jose Cardel and Agua Dulce) showed altered enzyme activity for $\alpha$-esterases (' $50 \%$ of the individuals exceeded the threshold of resistance), and Hoctun showed incipient altered enzyme activity (39\% of the individuals exceeded the threshold). Merida, Agua Dulce and Jose Cardel showed altered enzyme activity for $\beta$-esterases (' $50 \%$ ), and Progreso, Hunucma and Hoctun showed incipient enzyme alteration with percentages ranging between $16-43 \%$. Altered activity of MFOs was detected in Progreso, Hoctun, Agua Dulce and José Cardel in 69-95\% of the specimens analyzed. Only the Agua Dulce population showed altered enzyme activity for GSTs (71\%) in the parental generation.

The results show variations in enzyme activity across populations over the generations post-selection. However, when comparing $\mathrm{F}_{\mathrm{S} 5}$ vs $\mathrm{F}_{\mathrm{S} 0}$, $\alpha$-esterases remained altered in the Progreso, Hunucma and Agua Dulce; $\beta$-esterases in Progreso and Agua Dulce, and an increase in the mean activity levels of GSTs was observed only in the Hoctun and Agua Dulce populations $(P<0.0001)$ (Table 10$)$. GSTs were the only enzyme family for which a significant correlation was detected between level of activity and $\mathrm{LC}_{50}$ values, but only in the $\mathrm{F}_{\mathrm{S} 4}$ generation $(r=0.83, P<0.05)$.

\section{Discussion}

Target-site insensitivity and increased metabolic activity are key mechanisms of pyrethroid resistance in mosquito populations worldwide. The control of adult mosquitoes in Mexico has been carried out mainly with pyrethroids since 1999, and deltamethrin has been in use since the beginning of 2000 [9]. In the present study, five of the populations analyzed were found to be highly resistant to the pyrethroid deltamethrin in the parental generation according to the $\mathrm{LC}_{50}\left(\mathrm{RRLC}_{50}>10\right.$-fold), and only 


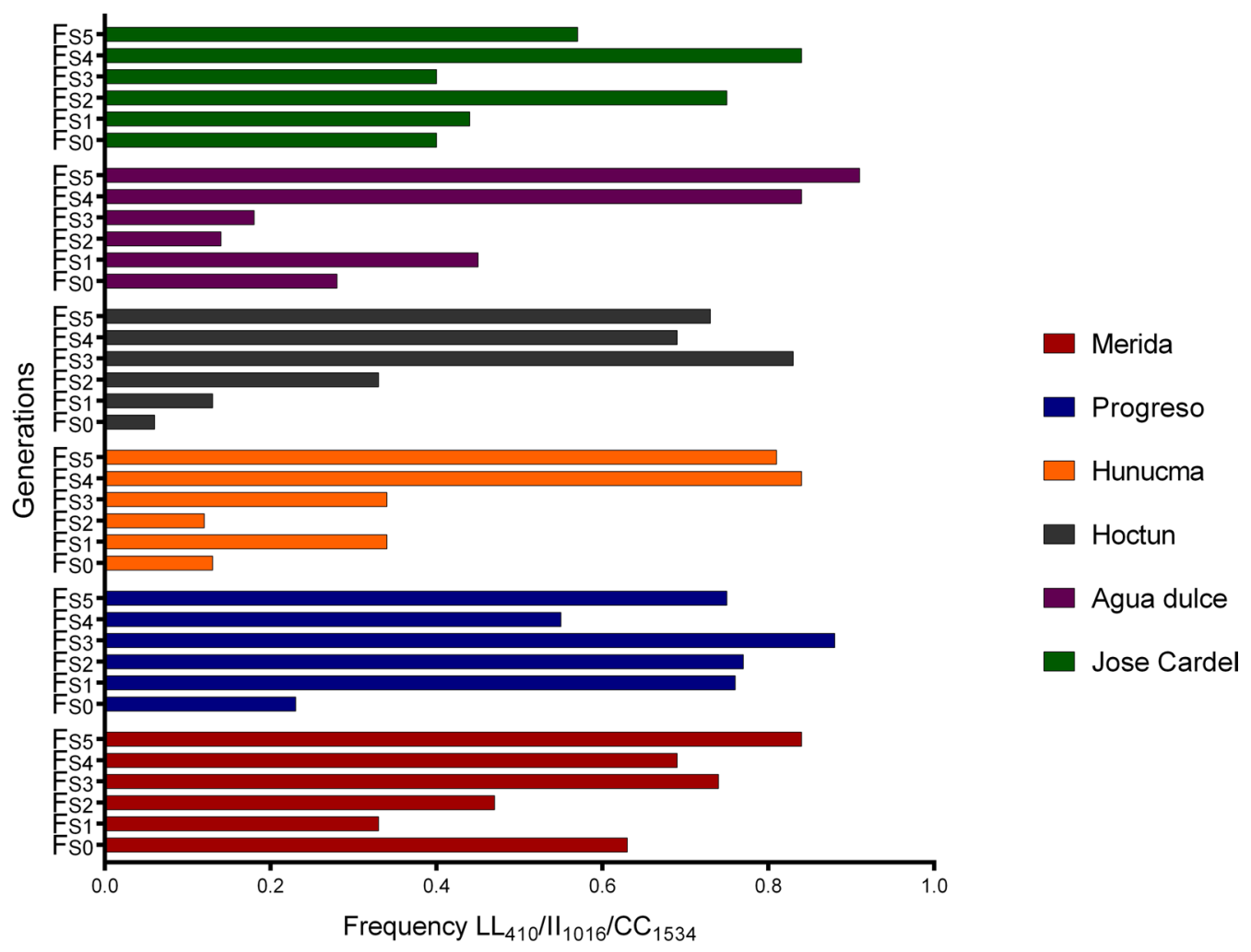

Fig. 4 Frequencies of the resistant trilocus genotype $\mathrm{LL}_{410} / \mathrm{II}_{1,016} / \mathrm{CC}_{1,534}$ across selection with deltamethrin $\left(\mathrm{F}_{50}-\mathrm{F}_{51}\right)$ from six populations of Aedes aegypti from Mexico

one population showed moderate resistance $\left(\mathrm{RRCL}_{50}\right.$ 5-10-fold). Likewise, high $\mathrm{RRKC}_{50}$ values were detected in two populations, three showed moderate resistance, and one showed low resistance. These results suggest that deltamethrin resistance is well-established in these populations.

In response to further deltamethrin selection, both $\mathrm{LC}_{50}$ and $\mathrm{KC}_{50}$ increased over the generations, with the concentrations capable of knocking down or killing 50\% of the population at least doubling by $\mathrm{F}_{\mathrm{S} 5}$ compared to $\mathrm{F}_{\mathrm{S} 0}$. The highest heritability values $\left(\mathrm{h}^{2} \geq 0.90\right)$ for $\mathrm{LC}_{50}$ were detected in the populations from Merida and Hoctun, followed by the Progreso population (0.61), suggesting high values of additive genetic variance. The highest value of $\mathrm{h}^{2}$ for $\mathrm{KC}_{50}$ was detected in the Progreso population $\left(\mathrm{h}^{2}>0.70\right)$, followed by the Merida population $(0.52)$. A high $h^{2}$ value predicts a rapid response to selection, while a low value suggests a slow response to artificial selection [46].

The frequencies of the L410, I1016 and C1534 alleles increased with deltamethrin selection in all populations. When analyzing the frequencies in the last selected generation among the different populations, the frequencies of the L410 allele ranged between $0.80-0.98$; this allele is able to significantly decrease the sensitivity of the sodium channel for both permethrin and deltamethrin [27]. The I1016 allele frequencies were $0.88-0.96$ in $\mathrm{F}_{\mathrm{S} 5}$ and the $\mathrm{C} 1534$ allele reached fixation by $\mathrm{F}_{\mathrm{S} 5}$ in all populations analyzed. These results suggest that deltamethrin can select for C1534 more rapidly than I1016, corroborating the findings of Alvarez et al. [60].

The co-occurrence of I1016 and C1534 and high levels of pyrethroid resistance has been reported in other countries [61-66]. In Mexico, the co-occurrence of these two mutations and pyrethroid resistance has been also documented [34, 50]. Vera-Maloof et al. [34] originally suggested that in order to present resistance to pyrethroids, the sequential evolution of both mutations was necessary. They considered it unlikely that I1016 had evolved independently due to the low fitness exhibited by haplotype I1016/F1534; instead, they hypothesized that C1534 first evolved by conferring a low level of resistance individually and that I1016 arose from haplotype V1016/C1534 and selected quickly due to the high level of resistance conferred by the double mutant haplotype. The sequential selection of F1534C and V1016I was later confirm by Chen et al. [31]. 


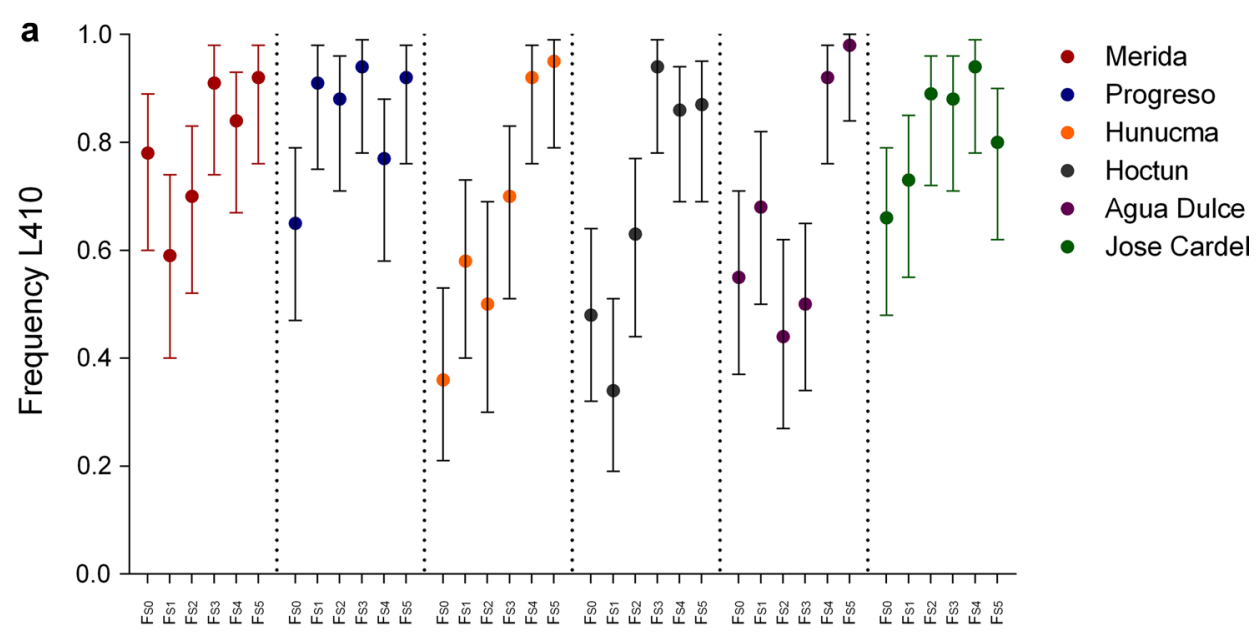

Generation
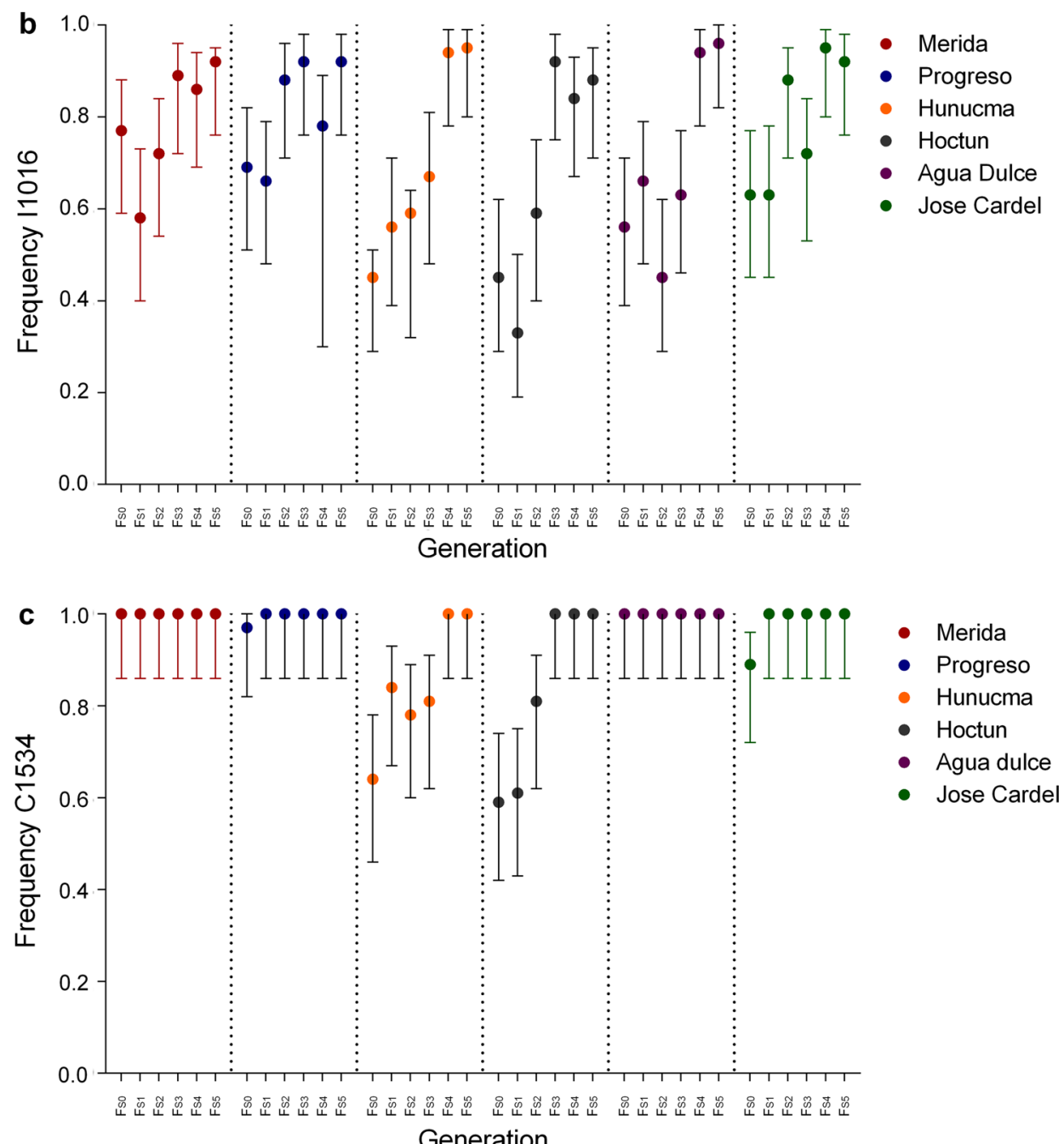

Fig. 5 Allele frequencies of $\mathrm{L} 410$ (a), 11016 (b) and C1534 (c) and their maximum and minimum frequencies in six Aedes aegypti populations across selection with deltamethrin $\left(\mathrm{F}_{S 0}-\mathrm{F}_{S 5}\right)$ 

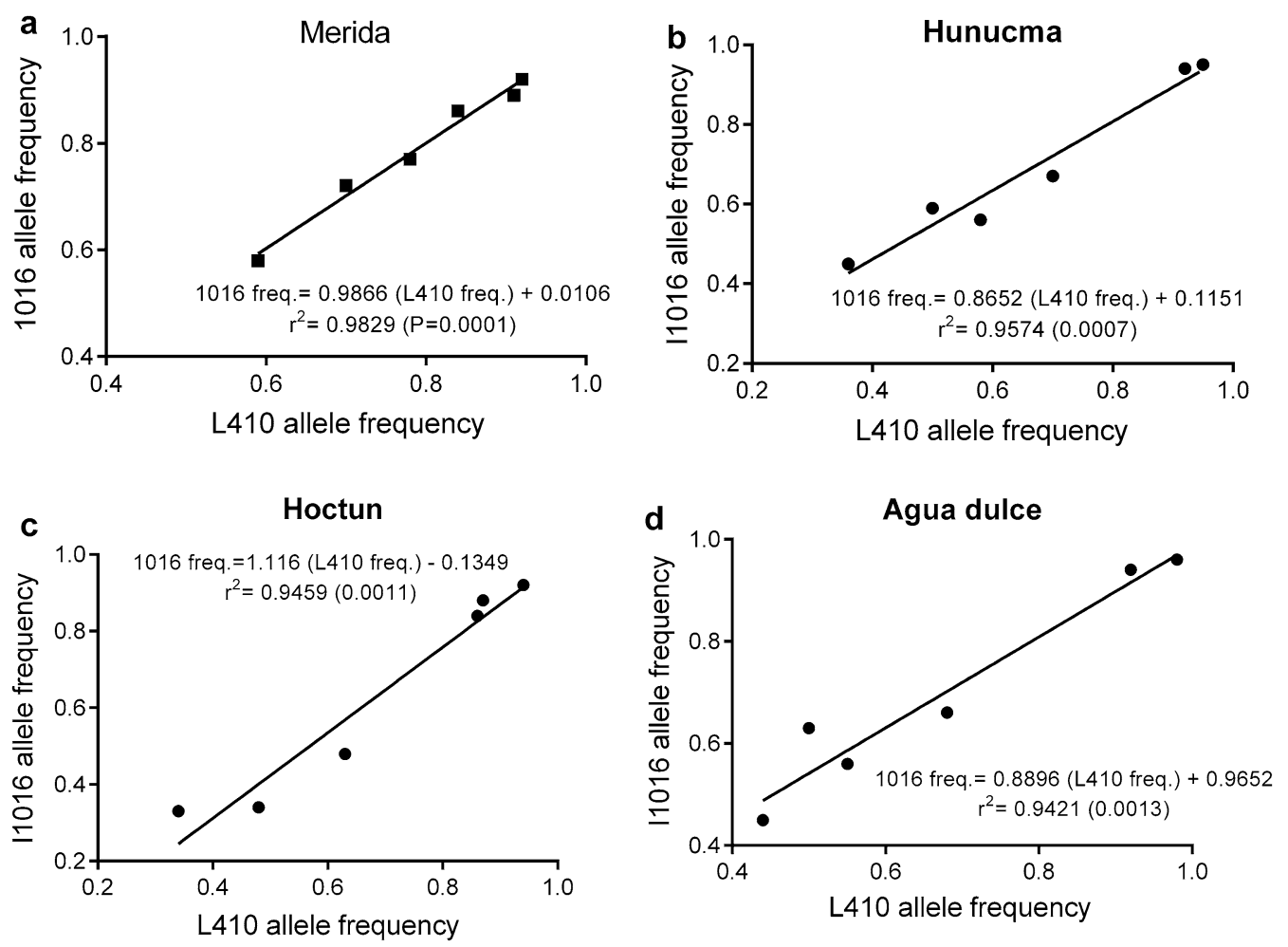

Fig. 6 Regression analysis of allele frequencies of two para mutations: 11016 vs $L 410$ across the selection with deltamethrin $\left(F_{50}-F_{S 5}\right)$ in Aedes aegypti from Merida (a), Hunucma (b), Hoctun (c), Agua dulce (d)

The V410L mutation was reported for the first time in the sodium channels of mosquitoes by Haddi et al. [27]. They demonstrated that this mutation reduced the sensitivity of the $v g s c$ gene to both type I (i.e. permethrin) and type II pyrethroids (i.e. deltamethrin). The presence of this mutation has been demonstrated in Ae. aegypti from Mexico for more than 16 years and it has been shown that L410 is in greater linkage disequilibrium with I1016 than with C1534. Our results show that L410 and I1016 responded to the selection with deltamethrin in a similar way in populations where C1534 was fixed; or in Hoctun and Hunucma where the increase in $\mathrm{C} 1534$ was associated with the increase in L410 and I1016. This is consistent with the proposed sequential model where both V410L and V1016I might have occurred independently on a C1534 haplotype and then became cis to $\mathrm{C} 1534$ by recombination. Or alternatively and considering that the F1534C mutation was fixed in practically four of the six basal populations $\left(\mathrm{F}_{\mathrm{S} 0}\right)$, the three mutations arose independently at very low frequencies, and then by two recombination events, came to occur in a cis arrangement [30].

Increased activity of mixed-function oxidases (MFOs) and esterase activity have been associated with pyrethroid resistance in Ae. aegypti [38, 67]. Our results detected incipiently altered levels of MFO activity in four populations. Altered levels of $\alpha$-esterase activity were detected in five populations, and at least four populations showed altered levels of $\beta$-esterase activity. However, there was no association between increased activities of these enzymes and phenotypic resistance, suggesting that these enzymes are not strongly associated with the metabolism of deltamethrin. However, contrary to the findings of Son et al. [68], who reported increased levels of MFOs in response to deltamethrin selection, we found that MFO activity decreased with successive selection. Given that the frequencies of the $k d r$ mutations increased significantly with deltamethrin selection, the rapid selection of these mutations could have conditioned the activity of the enzymes in relation to deltamethrin resistance.

One criticism in our methodology is that we compare the enzymatic activity of each generation of deltamethrin in each of the populations with respect to the enzymatic activity of the susceptible New Orleans strain, when the most appropriate comparison should be with respect to the same population, in the same generation, without selection with deltamethrin.

In the metabolic resistance, three families of detoxifying enzymes, oxidases, esterases and glutathione transferases are mainly involved, all associated to confer 


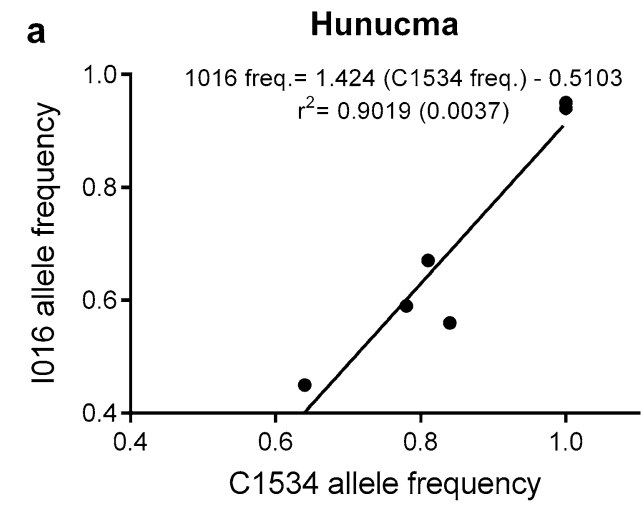

C

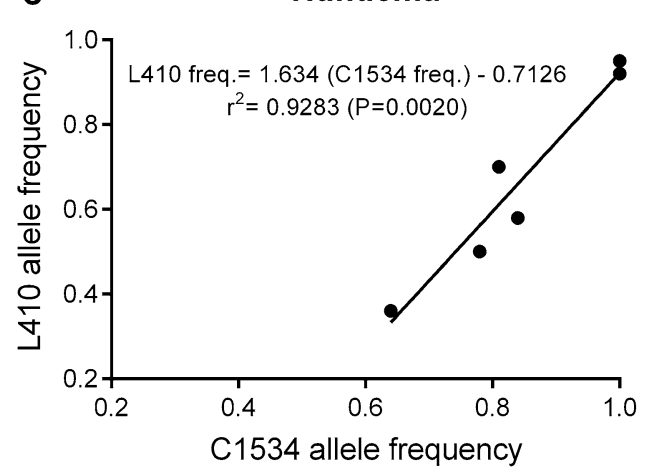

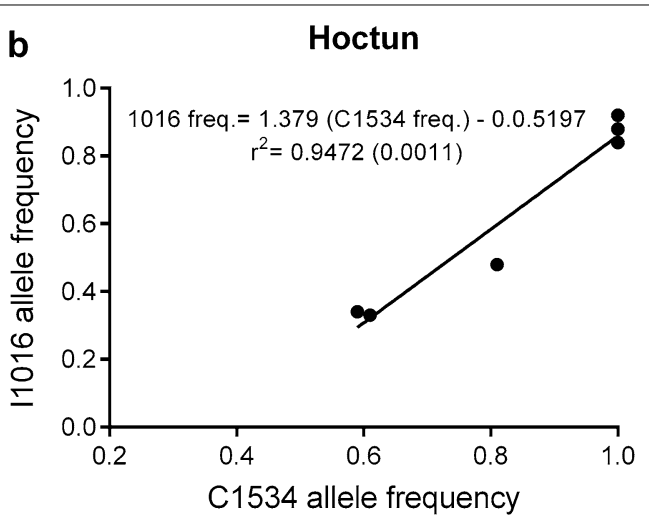

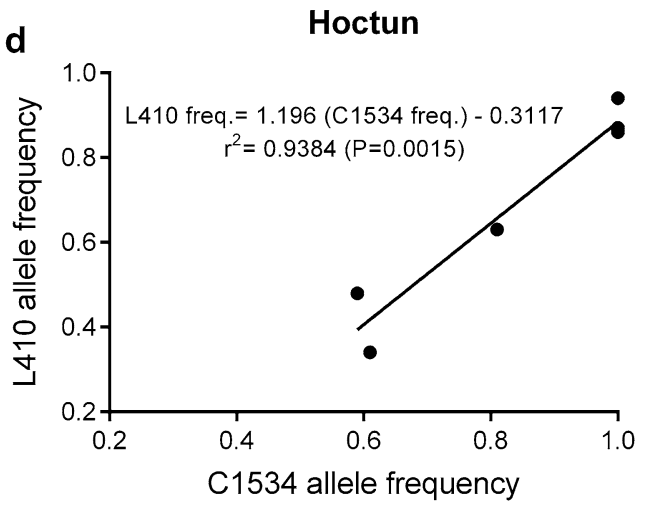

Fig. 7 Regression analysis of allele frequencies of C1534 vs $1016(\mathbf{a}, \mathbf{b})$ and C1534 vs $L 410(\mathbf{b}, \mathbf{c})$ across the selection with deltamethrin $\left(F_{S 0}-F_{S 5}\right)$ in Aedes aegypti from Hunucma and Hoctun

Table 9 Pearson's correlation coefficients ( $r$ ) (P-values in parenthesis) between the resistant allelic frequencies for L410, I1016, C1534 and with the frequencies of tri-locus genotypes vs $\mathrm{KC}_{50}$ and $\mathrm{LC}_{50}$ across the generations of selection with deltamethrin in five populations of Ae. aegypti from Mexico

\begin{tabular}{|c|c|c|c|c|c|c|}
\hline \multirow[t]{2}{*}{$\mathrm{KC}_{50} / \mathrm{LC}_{50}$} & \multicolumn{6}{|l|}{ Populations } \\
\hline & Merida & Progreso & Hunucma & Hoctun & Agua dulce & Jose Cardel \\
\hline \multicolumn{7}{|l|}{$\llcorner 410$} \\
\hline $\mathrm{KC}_{50}$ & $0.8303(P=0.040)$ & ns & ns & $0.8090(P=0.050)$ & ns & ns \\
\hline $\mathrm{LC}_{50}$ & $0.8323(P=0.039)$ & ns & $0.9506(P=0.004)$ & ns & ns & ns \\
\hline \multicolumn{7}{|l|}{11016} \\
\hline $\mathrm{KC}_{50}$ & $0.8459(P=0.033)$ & ns & ns & $0.8305(P=0.040)$ & ns & $0.8824(P=0.020)$ \\
\hline $\mathrm{LC}_{50}$ & $0.8410(P=0.036)$ & ns & $0.9249(P=0.008)$ & ns & ns & ns \\
\hline \multicolumn{7}{|l|}{ C1534 } \\
\hline $\mathrm{KC}_{50}$ & - & ns & ns & ns & - & ns \\
\hline $\mathrm{LC}_{50}$ & - & ns & $0.9023(P=0.014)$ & ns & - & $0.8401(P=0.036)$ \\
\hline \multicolumn{7}{|l|}{$\mathrm{LL}+\mathrm{II}+\mathrm{CC}$} \\
\hline $\mathrm{KC}_{50}$ & $0.8678(P=0.0251)$ & ns & ns & ns & ns & ns \\
\hline $\mathrm{LC}_{50}$ & - & ns & $0.8255(P=0.043)$ & ns & ns & ns \\
\hline
\end{tabular}


Table 10 Quantification of enzymatic activity in Aedes aegypti populations in the parental generation and deltamethrin-selected generations with respect to the New Orleans reference strain

\begin{tabular}{|c|c|c|c|c|c|c|c|c|c|c|}
\hline & \multirow{2}{*}{\multicolumn{3}{|c|}{$\frac{\text { a-esterases }}{(\mathrm{nmol} / \mathrm{mg} \mathrm{ptn} / \mathrm{min})}$}} & \multirow{2}{*}{\multicolumn{2}{|c|}{$\frac{\beta \text {-esterases }}{(\mathrm{nmol} / \mathrm{mg} \mathrm{ptn} / \mathrm{min})}$}} & \multirow{2}{*}{\multicolumn{2}{|c|}{$\frac{\text { MFO }}{\text { ( } \mu \mathrm{g} \text { Cyt/mg de ptn) }}$}} & \multirow{2}{*}{\multicolumn{2}{|c|}{$\frac{\text { GST }}{(\mathrm{nmol} / \mathrm{mg} \mathrm{ptn} / \mathrm{min})}$}} \\
\hline \multirow{2}{*}{\multicolumn{2}{|c|}{ Populatıon/generatıon }} & & & & & & & & & \\
\hline & & \multirow{2}{*}{$\frac{n}{30}$} & \multirow{2}{*}{$\begin{array}{l}\text { Mean } \pm \text { SD } \\
3.53 \pm 0.50\end{array}$} & \multirow{2}{*}{$\frac{\%>p 99^{c}}{4.77^{b}}$} & \multirow{2}{*}{$\begin{array}{l}\text { Mean } \pm \text { SD } \\
8.56 \pm 0.97\end{array}$} & \multirow{2}{*}{$\frac{\%>p 99}{10.9^{b}}$} & \multirow{2}{*}{$\begin{array}{l}\text { Mean } \pm S D \\
72.59 \pm 9.63\end{array}$} & \multirow{2}{*}{$\begin{array}{l}\%>p 99 \\
94.31^{b}\end{array}$} & \multirow{2}{*}{$\begin{array}{l}\text { Mean } \pm \text { SD } \\
0.05 \pm 0.01\end{array}$} & \multirow{2}{*}{$\frac{\%>p 99}{0.09^{b}}$} \\
\hline & New Orleans ${ }^{a}$ & & & & & & & & & \\
\hline \multirow{6}{*}{$\mathrm{F}_{0}$} & Merida & 30 & $7.18 \pm 1.21$ & 99 & $13.11 \pm 2.26$ & 79 & $87.30 \pm 16.44$ & 31 & $0.03 \pm 0.01$ & 0 \\
\hline & Progreso & 30 & $5.31 \pm 0.64$ & 82 & $10.00 \pm 1.03$ & 16 & $113.2 \pm 22.45$ & 69 & $0.08 \pm 0.03$ & 48 \\
\hline & Hunucma & 30 & $6.23 \pm 0.73$ & 99 & $10.95 \pm 0.80$ & 43 & $85.08 \pm 12.84$ & 23 & $0.05 \pm 0.01$ & 1 \\
\hline & Hoctún & 30 & $4.80 \pm 0.69$ & 39 & $10.76 \pm 1.10$ & 40 & $106.20 \pm 11.04$ & 81 & $0.07 \pm 0.01$ & 8 \\
\hline & Agua dulce & 30 & $6.58 \pm 0.85$ & 100 & $11.11 \pm 0.86$ & 54 & $132.10 \pm 30.02$ & 97 & $0.11 \pm 0.03$ & 71 \\
\hline & Cardel & 30 & $7.77 \pm 0.88$ & 100 & $12.75 \pm 1.56$ & 95 & $128.10 \pm 16.51$ & 95 & $0.06 \pm 0.01$ & 3 \\
\hline \multirow{6}{*}{$F_{1}$} & Merida & 30 & $5.73 \pm 0.69$ & 88 & $10.33 \pm 0.78$ & 24 & $94.27 \pm 10.83$ & 50 & $0.47 \pm 0.12$ & 0 \\
\hline & Progreso & 30 & $7.86 \pm 0.64$ & 100 & $9.81 \pm 1.12$ & 11 & $98.54 \pm 13.80$ & 56 & $0.63 \pm 0.27$ & 18 \\
\hline & Hunucma & 30 & $7.80 \pm 0.84$ & 100 & $10.02 \pm 1.28$ & 18 & $112.57 \pm 26.94$ & 70 & $0.89 \pm 0.28$ & 68 \\
\hline & Hoctún & 30 & $4.04 \pm 0.84$ & 11 & $9.43 \pm 1.71$ & 15 & $89.01 \pm 18.30$ & 38 & $0.34 \pm 0.37$ & 1 \\
\hline & Agua dulce & 30 & $6.69 \pm 0.78$ & 100 & $11.35 \pm 1.69$ & 57 & $107.46 \pm 13.62$ & 87 & $0.11 \pm 0.64$ & 6 \\
\hline & Cardel & 30 & $3.80 \pm 0.88$ & 10 & $8.11 \pm 1.07$ & 0 & $96.22 \pm 15.30$ & 50 & $0.87 \pm 0.30$ & 50 \\
\hline \multirow[t]{6}{*}{$\mathrm{F}_{2}$} & Merida & 30 & $6.75 \pm 0.60$ & 99 & $11.23 \pm 1.26$ & 74 & $91.57 \pm 11.82$ & 33 & $0.70 \pm 0.15$ & 25 \\
\hline & Progreso & 30 & $6.52 \pm 0.87$ & 99 & $12.44 \pm 1.30$ & 89 & $87.45 \pm 14.30$ & 32 & $0.54 \pm 0.18$ & 3 \\
\hline & Hunucma & 30 & $6.03 \pm 0.82$ & 96 & $10.51 \pm 0.66$ & 79 & $82.38 \pm 7.83$ & 7 & $0.37 \pm 0.11$ & 0 \\
\hline & Hoctún & 30 & $6.27 \pm 0.47$ & 99 & $10.84 \pm 0.98$ & 44 & $86.52 \pm 12.45$ & 31 & $0.87 \pm 0.27$ & 58 \\
\hline & Agua dulce & 30 & $6.69 \pm 0.79$ & 99 & $9.45 \pm 1.16$ & 5 & $81.67 \pm 11.91$ & 6 & $0.73 \pm 0.29$ & 31 \\
\hline & Cardel & 30 & $6.60 \pm 0.65$ & 99 & $12.87 \pm 1.15$ & 94 & $95.78 \pm 14.69$ & 39 & $0.32 \pm 0.11$ & 0 \\
\hline \multirow[t]{6}{*}{$\mathrm{F}_{3}$} & Merida & 30 & $4.43 \pm 5.22$ & 64 & $9.93 \pm 1.16$ & 20 & $79.41 \pm 12.43$ & 10 & $0.56 \pm 0.21$ & 17 \\
\hline & Progreso & 30 & $6.97 \pm 0.45$ & 99 & $11.70 \pm 0.93$ & 81 & $84.93 \pm 7.03$ & 9 & $1.13 \pm 0.26$ & 90 \\
\hline & Hunucma & 30 & $8.80 \pm 0.56$ & 99 & $13.85 \pm 1.05$ & 99 & $76.67 \pm 7.06$ & 0 & $0.54 \pm 0.12$ & 3 \\
\hline & Hoctún & 30 & $6.21 \pm 0.39$ & 99 & $10.50 \pm 0.69$ & 33 & $73.27 \pm 4.90$ & 0 & $0.42 \pm 0.11$ & 0 \\
\hline & Agua dulce & 30 & $6.51 \pm 0.85$ & 99 & $10.89 \pm 1.67$ & 44 & $83.45 \pm 10.15$ & 5 & $0.13 \pm 0.03$ & 0 \\
\hline & Cardel & 30 & $7.21 \pm 0.60$ & 99 & $11.17 \pm 0.90$ & 49 & $77.59 \pm 6.37$ & 0 & $0.50 \pm 0.14$ & 1 \\
\hline \multirow[t]{6}{*}{$\mathrm{F}_{4}$} & Merida & 30 & $4.59 \pm 0.67$ & 29 & $10.19 \pm 1.78$ & 20 & $87.68 \pm 8.14$ & 22 & $0.90 \pm 0.21$ & 75 \\
\hline & Progreso & 30 & $3.20 \pm 0.38$ & 0 & $6.72 \pm 0.60$ & 0 & $95.73 \pm 6.40$ & 57 & $0.61 \pm 0.52$ & 34 \\
\hline & Hunucma & 30 & $5.57 \pm 0.69$ & 90 & $8.51 \pm 0.88$ & 0 & $84.16 \pm 10.74$ & 19 & $0.64 \pm 0.25$ & 22 \\
\hline & Hoctún & 30 & $5.34 \pm 0.61$ & 82 & $10.17 \pm 0.90$ & 25 & $82.48 \pm 8.22$ & 19 & $0.49 \pm 0.16$ & 1 \\
\hline & Agua dulce & 30 & $5.55 \pm 0.57$ & 94 & $9.52 \pm 0.86$ & 2 & $75.84 \pm 8.56$ & 0 & $0.50 \pm 0.18$ & 3 \\
\hline & Cardel & 30 & $9.45 \pm 0.83$ & 99 & $14.67 \pm 1.32$ & 97 & $70.20 \pm 9.79$ & 0 & $0.65 \pm 0.23$ & 10 \\
\hline \multirow[t]{6}{*}{$\mathrm{F}_{5}$} & Merida & 30 & $4.29 \pm 0.55$ & 14 & $7.53 \pm 0.97$ & 0 & $61.85 \pm 8.80$ & 0 & $0.59 \pm 0.18$ & 17 \\
\hline & Progreso & 30 & $5.84 \pm 0.68$ & 92 & $12.22 \pm 1.17$ & 91 & $72.17 \pm 7.59$ & 0 & $0.78 \pm 0.30$ & 47 \\
\hline & Hunucma & 30 & $7.05 \pm 0.55$ & 99 & $10.76 \pm 0.58$ & 34 & $70.72 \pm 7.03$ & 0 & $0.45 \pm 0.11$ & 0 \\
\hline & Hoctún & 30 & $3.68 \pm 0.50$ & 1 & $8.19 \pm 1.19$ & 0 & $71.93 \pm 6.28$ & 0 & $0.86 \pm 0.20$ & 54 \\
\hline & Agua dulce & 30 & $6.69 \pm 0.81$ & 99 & $11.28 \pm 1.40$ & 55 & $67.35 \pm 6.25$ & 0 & $1.03 \pm 0.35$ & 80 \\
\hline & Cardel & 30 & $4.78 \pm 0.81$ & 49 & $10.27 \pm 1.22$ & 29 & $77.07 \pm 6.16$ & 0 & $0.49 \pm 0.17$ & 2 \\
\hline
\end{tabular}

Abbreviations: $\mathrm{n}$, sample size; $\mathrm{SD}$, standard deviation

a New Orleans: susceptible reference strain

b p99, 99th percentile for reference strains

c $\%>$ p99 percentage of individuals above 99 th percentile of reference strain, values classified as altered (> 50\%) are in bold

resistance through overexpression, increased insecticide metabolism or a greater affinity for the chemical $[6,17]$. An example of this contribution is reported by Lumjuan et al. [69] who determined an overexpression of GSTe2, GSTe5, GSTe7 and GSTE5-5 of epsilon glutathione transferase in individuals with resistance to DDT and pyrethroids. This fact is directly associated with the reduction in the susceptibility of these individuals to 
chemical control. Similarly, using quantitative trait loci (QTL) it was shown that temephos resistance is related to a QTL on chromosome two where a carboxylesterase cluster occurs; subsequent studies showed that there is an increase in the expression of these enzymes and therefore a possible intervention as a resistance mechanism [70]. Similar results were reported by Saavedra et al. [71], where the significant participation of an esterase marker discovered by QTL, CCEunk7o, and its relationship to resistance development in conjunction with mutations in the voltage-gated sodium channel was demonstrated.

Alongside gene expression studies, several investigations focus on studying the levels of detoxifying enzymatic activity. Proof of this is the increase in activity suffered by multiple-function oxidases, which includes cytochrome P450, in individuals selected with deltamethrin for 15 generations compared to the unselected susceptible group [60]; however, as for this mechanism to be in itself the one that confers insecticide resistance is questionable since other mechanisms were described for these populations.

To verify that the enzymatic activity has a direct effect on the decrease in susceptibility, synergists are used, chemical compounds that inhibit detoxifying enzymes. By inhibiting the enzymatic activity of multiple function oxidases, esterases and glutathione transferases directly involved as a resistance mechanism, proof of this is reported by Bharati \& Saha [72] where an increase in enzymatic activities of multiple-function oxidases (CYP450) and carboxylesterases was identified as a mechanism of resistance, by inhibiting such enzymes, the susceptibility to the pyrethroid deltamethrin and the carbamate propoxur was recovered in populations of $A e$. aegypti from Bangladesh. A similar pattern was demonstrated in populations of Ae. aegypti resistant to DDT and pyrethroids from Selangor, Malaysia, where the inhibition of multiple function oxidases and glutathione-S transferases through an assay with synergists correlated with an increase in the activity of such enzymes as a possible mechanism of resistance [73].

Identifying the direct and exclusive participation of metabolic resistance to insecticides is a complex process due to the simultaneous presence of mechanisms such as target site mutations and elevated levels of detoxifying enzymes. Despite this, the occurrence of Ae. albopictus populations free of insecticide-resistant target site mutations has been reported, demonstrating the direct and exclusive participation of cytochrome P450 genes such as CYP6P12 and the overexpression of cuticular genes as the main resistance mechanisms [74].

\section{Conclusions}

The high levels of resistance associated with high frequencies of $k d r$ alleles L410, I1016 and C1534 obtained through artificial selection suggest the important role of these mutations in resistance to deltamethrin. The role of metabolic resistance was less clear, as the activity levels of key enzyme groups appeared to increase or decrease without clearly relating to the selection with deltamethrin. These findings highlight the importance of monitoring the susceptibility status of mosquito populations before choosing insecticide products for vector control, especially in areas where resistance may already be present and further selection could quickly result in fixation of $k d r$ mutations.

\section{Abbreviations}

kdr: knockdown resistance; $\mathrm{KC}_{50}: 50 \%$ knockdown concentration; $\mathrm{LC}_{50}: 50 \%$ lethal concentration; RR: resistance ratio; vgsc: voltage gated sodium channel; CDC: Centers for Disease Control and Prevention; WHO: World Health Organization; DDT: dichlorodiphenyltrichloroethane; PCR: polymerase chain reaction; MFOs: mixed-function oxidases; GSTs: glutathione S-transferases.

\section{Acknowledgements}

We would like to thank Kevin Ontiveros-Zapata (Universidad Autonoma de Nuevo Leon, Mexico) for processing mosquitoes in the laboratory.

\section{Disclaimer}

The findings and conclusions in this paper are those of the authors and do not necessarily represent the official position of the Centers for Disease Control and Prevention.

\section{Authors' contributions}

AEF conceived the study and obtained funding. YCP, PMS and GPG carried out collections of the populations included in the study. YCP performed the laboratory work. AEF, YCP, AL, GPG, KVS, BLM and IPRS analyzed the data. AEF $Y C P$ and $A L$ wrote the manuscript. All authors read and approved the final manuscript.

\section{Funding}

This study was financially supported by CONACYT Mexico through the fund Problemas Nacionales, Project no. PN2016-2134.

Availability of data and materials

All data generated or analyzed during this study are included in the published article.

\section{Ethics approval and consent to participate}

Not applicable.

\section{Consent for publication}

Not applicable.

\section{Competing interests}

The authors declare that they have no competing interests.

\footnotetext{
Author details

${ }^{1}$ Facultad de Ciencias Biologicas, Universidad Autonoma de Nuevo Leon, Cd, Universitaria, San Nicolas de los Garza, N.L. CP. 66455, Mexico. ${ }^{2}$ Division of Parasitic Diseases and Malaria, Centers for Disease Control and Prevention, Atlanta, GA, USA. ${ }^{3}$ Unidad Colaborativa para Bioensayos Entomologicos, Universidad Autonoma de Yucatan, Campus de Ciencias Biologicas y Agropecuarias, Merida, Yucatan, Mexico.
} 
Received: 2 January 2020 Accepted: 24 April 2020

Published online: 06 May 2020

\section{References}

1. Smith L, Kasai S, Scott JG. Pyrethroid resistance in Aedes aegypti and Aedes albopictus: important mosquito vectors of human diseases. Pestic Biochem Physiol. 2016;133:1-12

2. Huber JH, Childs ML, Caldwell JM, Mordecai EA. Seasonal temperature variation influences climate suitability for dengue, chikungunya, and Zika transmission. Plos Neglect Trop Dis. 2018;12:e0006451.

3. Kandel Y, Vulcan J, Rodriguez SD, Moore E, Chung HN, Mitra S, et al. Widespread insecticide resistance in Aedes aegypti L. from New Mexico, USA. PLoS One. 2019;14:e0212693.

4. WHO. WHO recommended insecticides for indoor residual spraying against malaria vectors. Geneva: World Health Organization; 2018. https:// www.who.int/neglected_diseases/vector_ecology/vector-control/Insec ticides_IRS_22_September_2018.pdf?ua=1. Accessed 19 Feb 2020.

5. WHO. Global strategy for dengue prevention and control 2012-2020. Geneva: World Health Organization; 2012. https://www.afro.who.int/ publications/global-strategy-dengue-prevention-and-control-2012-2020. Accessed 19 Feb 2020.

6. Moyes C, Vontas J, Martins A, Ng L, Koou SY, Dusfour I. Contemporary status of insecticide resistance in the major Aedes vectors of arboviruses infecting humans. PLoS Neglect Trop Dis. 2017;11:e0005625.

7. Amelia-Yap ZH, Chen CD, Sofian-Azirun MM, Low VL. Pyrethroid resistance in the dengue vector Aedes aegypti in Southeast Asia: present situation and prospects for management. Parasit Vectors. 2018;11:332.

8. Ray DE, Fry JR. A reassessment of the neurotoxicity of pyrethroid insecticides. Pharmacol Therapeut. 2006;111:174-93.

9. DOF (Diario Oficial de la Federacion). NOM-032-SSA-2-2002 para la vigilancia epidemiológica, prevención y control de enfermedades transmitidas por vectores. Mexico. 2003. http://www.salud.gob.mx/unidades/ cdi/nom/032ssa202.html. Accessed 19 Feb 2020.

10. CENAPRECE. Lista de Insumos Recomendados por el CENAPRECE para el combate de insectos vectores 2019. Mexico. 2019. https://www.gob.mx/ cms/uploads/attachment/file/469289/Lista_de_Insumos_Recomendad os por el CENAPRECE.pdf. Accessed 19 Feb 2020.

11. Siller Q, Ponce G, Lozano S, Flores AE. Update on the frequency of Ile1016 mutation in voltage gated sodium channel gene of Aedes aegypti in Mexico. J Am Mosq Control Assoc. 2011:27:357-62.

12. Aponte HA, Penilla-Navarro P, Dzul-Manzanilla F, Che-Mendoza A, López AD, Solís F, et al. The pyrethroid resistance status and mechanisms in Aedes aegypti from Guerrero state, Mexico. Pest Biochem Phys. 2013:107:226-34

13. Flores AE, Ponce-García G, Lopez-Monroy B, Villanueva-Segura OK, Rodriguez-Sánchez IP, Arredondo-Jímenez JI, Manrique-Saide P. Current status of the insecticide resistance in Aedes aegypti (Diptera: Culicidae) from Mexico. In: Stanislav T, editor. insecticide resistance. London: IntechOpen; 2016. p. 99-109.

14. Flores AE, Grajales JS, Fernandez I, Ponce G, Loaiza H, Lozano S, et al. Mechanisms of insecticide resistance in field populations of Aedes aegypti (L.) from Quintana Roo, Southern Mexico. J Am Mosq Control. 2006:22:672-7.

15. Stevenson BJ, Pignatelli P, Nikou D, Paine MJ. Pinpointing P450s Associated with pyrethroid metabolism in the dengue vector, Aedes aegypti: developing new tools to combat insecticide resistance. PLoS Neglect Trop Dis. 2012;6:e1595.

16. Brogdon WG, MCAllister JC. Insecticide resistance and vector control. Emerg Infect Dis. 1998;4:605-13.

17. Liu N. Insecticide resistance in mosquitoes: impact, mechanisms, and research directions. Ann Rev Entomol. 2015;60:537-59.

18. Narashashi T. Neuronal ion channels as the target sites of insecticides. Pharmacol Toxicol. 1996;79:1-14.

19. Rinkevich FD, Hedtke SM, Leichter CA, Harris SA, Su C, Brady SG, et al. Multiple origins of $k d r$-type resistance in the house fly, Musca domestica. PLOS One. 2012:7:e52761.
20. Du Y, Nomura Y, Satar G, Hu Z, Nauen R, He SY, et al. Molecular evidence for dual pyrethroid-receptor sites on a mosquito sodium channel. Proc Natl Acad Sci USA. 2013;110:11785-90.

21. Brengues C, Hawkes NJ, Chandre F, McCarroll L, Duchon S, et al. Pyrethroid and DDT cross-resistance in Aedes aegypti is correlated with novel mutations in the voltage-gated sodium channel gene. Med Vet Entomol. 2003;17:87-94.

22. Saavedra-Rodriguez K, Urdaneta-Marquez L, Rajatileka S, Moulton M, Flores $A E$, Fernandez-Salas I, et al. A mutation in the voltage-gated sodium channel gene associated with pyrethroid resistance in Latin American Aedes aegypti. Insect Mol Biol. 2007;16:785-98.

23. Chang C, Shen WK, Wang TT, Lin YH, Hsu EL, et al. A novel amino acid substitution in a voltage-gated sodium channel is associated with knockdown resistance to permethrin in Aedes aegypti. Insect Mol Biol. 2009;39:272-8.

24. Srisawat R, Komalamisra N, Eshita Y, Zheng M, Ono K, et al. Point mutations in domain II of the voltage-gated sodium channel gene in deltamethrin-resistant Aedes aegypti (Diptera: Culicidae). Appl Entomol Zool. 2010;45:275-82

25. Yanola J, Somboon P, Walton C, Nachaiwieng W, Somwang P, Prapanthadara LA. High-throughput assays for detection of the F1534C mutation in the voltage-gated sodium channel gene in permethrin-resistant Aedes aegypti and the distribution of this mutation throughout Thailand. Trop Med Int Health. 2011;16:501-9.

26. Kushwah RBS, Dykes CL, Kapoor N, Adak T, Singh OP. Pyrethroid-resistance and presence of two knockdown resistance (kdr) mutations, F1534C and a novel mutation T1520I, in Indian Aedes aegypti. PLoS Neglect Trop Dis. 2015;9:e3332

27. Haddi K, Tome HVV, Du Y, Valbon WR, Nomura Y, Martins GF, et al. Detection of a new pyrethroid resistance mutation (V410L) in the sodium channel of Aedes aegypti: a potential challenge for mosquito control. Sci Rep. 2017;7:46549.

28. Granada Y, Mejia-Jaramillo A, Strode C, Triana-Chavez O. A point mutation V419L in the sodium channel gene from natural populations of Aedes aegypti is involved in resistance to $\lambda$-cyhalothrin in Colombia. Insects. 2018;9:E23.

29. Kushwah RBS, Kaur T, Dykes CL, Kumar RH, Kapoor N, Singh OP. A new knockdown resistance $(k d r)$ mutation F1534L in Aedes aegypti associated with insecticide resistance. BioRxiv. 2019;740829.

30. Saavedra-Rodriguez K, Maloof FV, Campbell CL, Garcia-Rejon J, Lenhart A, Penilla P, et al. Parallel evolution of vgsC mutations at domains IS6, IIS6 and IIIS6 in pyrethroid resistant Aedes aegypti from Mexico. Sci Rep. 2018;8:6747.

31. Chen M, Du Y, Wu S, Nomura Y, Zhu G, Zhorov BS, Dong K. Molecular evidence of sequential evolution of DDT- and pyrethroid-resistant sodium channel in Aedes aegypti. PLoS Neglect Trop Dis. 2019;13:e0007432.

32. Fernando SD, Hapugoda M, Perera R, Saavedra-Rodriguez K, Black WC, De Silva NK. First report of $\mathrm{V} 1016 \mathrm{G}$ and S989P knockdown resistant (kdr) mutations in pyrethroid-resistant Sri Lankan Aedes aegypti mosquitoes. Parasit Vectors. 2018;11:526

33. Hu Z, Du Y, Nomura Y, Dong K. A sodium channel mutation identified in Aedes aegypti selectively reduces cockroach sodium channel sensitivity to type I, but not type II pyrethroids. Insect Biochem Mol Biol. 2011:41:9-13.

34. Vera-Maloof FZ, Saavedra-Rodriguez K, Elizondo-Quiroga AE, LozanoFuentes S, Black WC IV. Coevolution of the Ile 1,016 and Cys1,534 mutations in the voltage gated sodium channel gene of Aedes aegypti in Mexico. PLoS Neglect Trop D. 2015;9:e0004263.

35. Ponce-Garcia G, Flores AE, Fernandez-Salas I, Saavedra-Rodriguez K Reyes-Solis G, Lozano-Fuentes S. Recent rapid rise of a permethrin knock down resistance allele in Aedes aegypti in Mexico. PLoS Neglect Trop Dis. 2009;3:e531.

36. Villanueva-Segura OK, Ontiveros-Zapata K, Lopez-Monroy B, PonceGarcia G, Gutierrez-Rodriguez SM, Davila-Barboza JA, et al. Distribution and frequency of the $k d r$ mutation $V 410 \mathrm{~L}$ in natural populations of Aedes aegypti (L.) (Diptera: Culicidae) from eastern and southern Mexico. J Med Entomol. 2020;57:218-23.

37. Hemingway J, Ranson $\mathrm{H}$. Insecticide resistance in insect vectors of human disease. Annu Rev Entomol. 2000;45:371-91. 
38. Flores AE, Abeldaño W, Fernandez I, Badii MH, Loaiza H, Ponce G, et al. Elevated a-esterase levels associated with permethrin tolerance in Aedes aegypti (L.) from Baja California, México. Pestic Biochem Phys. 2005;82:66-78.

39. Bisset JA, Marin R, Rodriguez MM, Severson DW, Ricardo Y, French L, Díaz M, Perez O. Insecticide resistance in two Aedes aegypti (Diptera: Culicidae) strains from Costa Rica. J Med Entomol. 2013;50:352-61.

40. Flores AE, Reyes G, Fernández I, Sánchez FJ, Ponce G. Resistance to permethrin in Aedes aegypti (L.) in northern Mexico. Southwest Entomol. 2009;34:167-77.

41. Lopez B, Ponce G, Gonzalez JA, Gutierrez SM, Villanueva OK, Gonzalez $G$, et al. Susceptibility to chlorpyrifos in pyrethroid-resistant populations of Aedes aegypti (Diptera: Culicidae) from Mexico. J Med Entomol. 2014;51:644-9.

42. Brogdon W, Chan A. Guidelines for evaluating insecticide resistance in vectors using the $C D C$ bottle bioassay. CDC technical report. Methods in Anopheles research, 2nd ed. Atlanta: Centers for Disease Control and Prevention; 2010.

43. Lozano FS, Saavedra RK, Black W IV, Eisen L. QCAL: a software application for the calculation of dose-response curves in insecticide resistance bioassays. J Am Mosq Control. 2012;28:59-91.

44. Abbott WS. A method of computing the effectiveness of an insecticide. Econ Entomol. 1925;18:265-7.

45. Mazzarri M, Georghiou G. Characterization of resistance to organophosphate, carbamate, and pyrethroid insecticides in field population of Aedes aegypti from Venezuela. J Am Mosq Control. 1995;11:315-22.

46. Saavedra- Rodriguez K, Flores AE, Fernandez I, Strode C, Ranson H, Hemingway J, Black WC IV. Transcription of detoxification genes after permethrin selection in the mosquito Aedes aegypti. Insect Mol Biol. 2012;21:61-77.

47. Tabashnik BE. Resistance risk assessment: realized heritability of resistance to Bacillus thuringiensis in diamondback moth (Lepidoptera: Plutellidae), tobacco budworm (Lepidoptera: Noctuidae), and Colorado potato beetle (Coleoptera: Chrysomelidae). J Econ Entomol. 1992:85:1551-9.

48. Falconer DS. Introduction to quantitative genetics. 3rd ed. Harlow, Essex, UK/New York: Longmans Green/John Wiley \& Sons; 1989.

49. Coen E, Strachan T, Dover G. Dynamics of concerted evolution of ribosomal DNA and histone gene families in the melanogaster species subgroup of Drosophila. J Mol Biol. 1982;158:17-35.

50. Lopez-Monroy B, Gutierrez-Rodriguez SM, Villanueva-Segura OK, Ponce-Garcia G, Morales-Forcada F, Alvarez LC, Flores AE. Frequency and intensity of pyrethroid resistance through the CDC bottle bioassay and their association with the frequency of $k d r$ mutations in Aedes aegypti (Diptera: Culicidae) from Mexico. Pest Manag Sci. 2018:74:2176-84

51. Wright S. Systems of mating. II. The effects of inbreeding on the genetic composition of a population. Genetics. 1921;6:124-43.

52. Agresti A, Coull BA. Approximate is better than "exact" for interval estimation of binominal proportions. Am Stat. 1998;52:1 19-26.

53. Brogdon WG. Biochemical resistance detection: an alternative to bioassay. Parasitol Today. 1989;5:56-60.

54. Brogdon WG, Barber AM. Microplate assay of glutathione S-transferase activity for resistance detection in single mosquito triturates. Comp Biochem Physiol. 1990;96:339-42.

55. Brogdon WG, MCAllister JC, Vulule J. Heme peroxidase activity measured in single mosquitoes identifies individuals expressing an elevated oxidase for insecticide resistance. J Am Mosq Control Assoc. 1997;13:233-7.

56. Bradford M. A rapid and sensitive method for the quantitation of microgram quantities of protein utilizing the principle of protein-dye binding. Anal Biochem. 1976;72:248-54.

57. Valle D, Montella IR, Ribeiro RA, Viana-Medeiros PF, Martins-Júnior AJ, Lima JBP. Metodologia para quantificação de atividade de enzimas relacionadas com a resistência a inseticidas em Aedes aegypti/Quantification methodology for enzyme activity related to insecticide resistance in Aedes aegypti. Rio de Janeiro, Brasília: Fundação Oswaldo Cruz, Secretaria de Vigilância em Saúde/Ministério da Saúde; 2006.
58. Hemingway J. Techniques to detect insecticide resistance mechanisms (field and laboratory manual) WHO/CDC/CPC/MAL/98.6. Geneva: World Health Organization; 1998.

59. Montella I, Martins A, Fernandez V, Pereira J, Braga I, Valle D. Insecticide resistance mechanisms of Brazilian Aedes aegypti populations from 2001 to 2004. Am J Trop Med Hyg. 2007;77:467-77.

60. Alvarez LC, Ponce G, Saavedra-Rodriquez K, Lopez B, Flores AE. Frequency of V1016l and F1534C mutations in the voltage-gated sodium channel gene in Aedes aegypti in Venezuela. Pest Manag Sci. 2015;71:863-9.

61. Kawada H, Oo SZ, Thaung S, Kawashima E, Maung YN, Thu HM, et al. Cooccurrence of point mutations in the voltage-gated sodium channel of pyrethroid-resistant Aedes aegypti populations in Myanmar. PLoS Neglect Trop Dis. 2014;8:e3032.

62. Chapadense FG, Fernandes EK, Lima JB, Martins AJ, Silva LC, Rocha WT, et al. Phenotypic and genotypic profile of pyrethroid resistance in populations of the mosquito Aedes aegypti from Goiânia, Central West Brazil. Rev Soc Bras Med Trop. 2015;48:607-9.

63. Dusfour I, Zorrilla P, Guidez A, Issaly J, Girod R. Deltamethrin resistance mechanisms in Aedes aegypti populations from three French overseas territories worldwide. PLoS Neglect Trop Dis. 2015;9:e0004226.

64. Maestre-Serrano R, Pareja-Loaiza P, Gomez-Camargo D, Ponce-García G, Flores AE. Co-occurrence of V1016l and F1534C mutations in the voltagegated sodium channel and resistance to pyrethroids in Aedes aegypti (L.) from the Colombian Caribbean region. Pest Manag Sci. 2019;75:1681-8.

65. Yaméogo F, Sanon A, McCall PJ, Shirozu T, Weetman D, et al. High frequencies of F1534C and V1016l kdr mutations and association with pyrethroid resistance in Aedes aegypti from Somgandé (Ouagadougou), Burkina Faso. Trop Med Health. 2019;47:4-11.

66. Deming R, Manrique-Saide P, Barreiro AM, Cardeña EU, Che-Mendoza $A$, Jones $B$, et al. Spatial variation of insecticide resistance in the dengue vector Aedes aegypti presents unique vector control challenges. Parasit Vectors. 2016;9:67.

67. Polson KA, Brogdon WG, Rawlins SC, Chadee DD. Characterization of insecticide resistance in Trinidadian strains of Aedes aegypti mosquitoes. Acta Trop. 2011;117:31-8.

68. Son-Un P, Choovattanapakorn N, Saingamsook J, Yanola J, Lumjuan N, et al. Effect of relaxation of deltamethrin pressure on metabolic resistance in a pyrethroid-resistant Aedes aegypti (Diptera: Culicidae) strain harboring fixed P989P and G1016G kdr alleles. J Med Entomol. 2018;55:975-81.

69. Lumjuan N, Rajatileka S, Changsom D, Wicheer J, Leelapat P, et al. The role of the Aedes aegypti Epsilon glutathione transferases in conferring resistance to DDT and pyrethroid insecticides. Insect Biochem Mol Biol. 2011:41:203-9.

70. Paiva MHS, Lovin DD, Mori A, Melo-Santos MAV, Severson DW, et al. Identification of a major quantitative trait locus determining resistance to the organophosphate temephos in the dengue vector mosquito Aedes aegypti. Genomics. 2016;107:40-8.

71. Saavedra-Rodriguez K, Strode C, Flores Suarez A, Fernandez Salas I, Ranson $\mathrm{H}$, et al. Quantitative trait loci mapping of genome regions controlling permethrin resistance in the mosquito Aedes aegypti. Genetics. 2008;180:1137-52.

72. Bharati M, Saha D. Multiple insecticide resistance mechanisms in primary dengue vector, Aedes aegypti (Linn.) from dengue endemic districts of sub-Himalayan West Bengal, India. PLoS One. 2018;13:e0203207.

73. Leong CS, Vythilingam I, Liew JWK, Wong ML, Wan-Yusof WS, et al. Enzymatic and molecular characterization of insecticide resistance mechanisms in field populations of Aedes aegypti from Selangor, Malaysia. Parasit Vectors. 2019:12:236.

74. Ishak IH, Riveron JM, Ibrahim SS, Stott R, Longbottom J, et al. The cytochrome P450 gene CYP6P12 confers pyrethroid resistance in kdr-free Malaysian populations of the dengue vector Aedes albopictus. Sci Rep. 2016;6:24707.

\section{Publisher's Note}

Springer Nature remains neutral with regard to jurisdictional claims in published maps and institutional affiliations. 